\title{
Mixed finite element - discontinuous finite volume element discretization of a general class of multicontinuum models
}

\author{
Ricardo Ruiz-Baier \\ Mathematical Institute, Oxford University, A. Wiles Building, \\ Radcliffe Observatory Quarter, Woodstock Road, OX2 6GG Oxford, UK \\ Ivan Lunati \\ Institute of Earth Sciences, University of Lausanne, Switzerland
}

\begin{abstract}
We present a novel discretization scheme tailored to a class of multiphase models that regard the physical system as consisting of multiple interacting continua. In the framework of mixture theory, we consider a general mathematical model that entails solving a system of mass and momentum equations for the mixture and for one of the phases. The model results in a strongly coupled and nonlinear system of partial differential equations that are written in terms of phase and mixture (barycentric) velocities, phase pressure, and saturation. We construct an accurate, robust and reliable hybrid method that combines a mixed finite element discretization of the momentum equations with a primal discontinuous finite volume-element discretization of the mass (or transport) equations. The scheme is devised for unstructured meshes and relies on mixed Brezzi-Douglas-Marini approximations of phase and total velocities, on piecewise constant elements for the approximation of phase or total pressures, as well as on a primal formulation that employs discontinuous finite volume elements defined on a dual diamond mesh to approximate scalar fields of interest (such as volume fraction, total density, saturation, etc.). As the discretization scheme is derived for a general formulation of multicontinuum physical systems, it can be readily applied to a large class of simplified multiphase models; on the other, the method can be seen as a generalization of these models that are commonly encountered in the literature and employed when the latter are not sufficiently accurate. An extensive set of numerical test cases involving two- and three-dimensional porous media are presented to demonstrate the accuracy of the method that displays an optimal convergence rate, the physics-preserving properties of the mixed-primal scheme, as well as the the robustness of the method, which is successfully applied to diverse physical phenomena such as density fingering, Terzaghi's consolidation, deformation of a cantilever bracket, and the Boycott effects. The applicability of the method is not limited to flow in porous media, but can be also employed to describe many other physical systems that are governed by a similar system of equations, e.g., to model multi-component materials.
\end{abstract}

Key words: Multiphase flow, Momentum and mass coupling, Mixture theory, Mixed finite element methods, Discontinuous finite volume-element methods 2000 MSC: 76S05, 65M60, 76T10

\section{Introduction}

Multiphase systems are encountered in many science and engineering applications, such as remediation of groundwater polluted by non-aqueous phase liquids, carbon capture and storage

Email addresses: ruizbaier@maths.ox.ac.uk (Ricardo Ruiz-Baier), ivan.lunati@unil.ch (Ivan Lunati) 
in geological formations, wastewater treatment, geomorphology and sediment transport, bioreactors and fluidized bed filters, formation of hydrates, triggering of landslides, exploitation of conventional and unconventional hydrocarbon resources, to name only a few.

The mathematical description of multiphase systems is intrinsically challenging because the governing conservation equations and the constitutive relations are highly nonlinear and characterized by strong coupling and stiff relaxation. Moreover, in many cases multiple spatial and temporal scales are present, and the phase properties display large contrasts. This makes it difficult to obtain accurate and efficient numerical methods that are able to describe the flow patterns and the velocity fields with the desired accuracy, and at the same time able to enforce physical characteristics of the solution such as local mass conservation, which is essential to avoid artificial sinks or sources. On the other hand, the approximations of the transport problem are required to satisfy a discrete maximum principle and to capture the sharp fronts associated to strong convection phenomena.

In the effort to propose techniques that display such features, in the last couple of decades an increasing number of studies have combined Finite Elements (FE) and Finite Volume (FV) methods to jointly exploit the intrinsic features of FE and FV-based schemes. Related literature includes the interaction of FE and continuous Finite Volume Element (FVE) methods applied to the study of Earth mantle convection [21, the hybrid (continuous) FVE - Discontinuous Finite Volume Element (DFVE) method proposed to solve the coupling of flow and transport in sedimentation problems [9], the mixed FE-FV methods introduced for two-phase flow in porous media [4, 28, the axisymmetric FE-FV scheme for variable density flows [11, pure FVE-based methods for two-phase flows [7, 25], and other similar discretizations [12, 24.

Here, we derive a discretization scheme for a very general formulation of multiphase flow in terms of mass and momentum conservation equations for the mixture and for one of the phases. (The formalism is presented for two phases, treated as interacting multicontinua, but can be easily extended to the case of an arbitrary number of phases, $N$, provided that the mass and momentum conservation equations of $N-1$ phases are considered in addition to those of the mixture). The interest in such formulation is twofold: on one hand, it allows us to readily discretize a large class of multi-continuum problems that are amenable to the archetypal model; on the other hand, the archetypal model provides a generalization of the particular models that can be used when the assumptions leading to the latter are not satisfied.

We propose to combine a mixed FE approximation of the momentum equations with a DFVE methods for the transport (mass conservation) equations. This is the first mixedprimal method stated for this general formulation and dealing with mass and momentum equations. Moreover, even if a number of hybrid FE-FV methods are available for multiphase flow problems, this is the first scheme that combines mixed FEM and DFVE. The primary reason to use a DFVE discretization is to incorporate the desirable properties of both FVE and discontinuous Galerkin (DG) methods, such as local conservation over the control volumes and the flexibility to choose tailored numerical fluxes as in finite volume (FV) methods (see e.g. 25]), which guarantees continuous fluxes across inter-element boundaries.

The spatial discretization of momentum equations is carried out with the help of a a dual diamond grid. The velocities are approximated with first order Brezzi-Douglas-Marini FE or with first order Raviart-Thomas elements, which ensure that fluxes are correctly approximated [20]; whereas the discretization of the scalar field variables is based upon piecewise linear DFVE approximations, which ensure local conservation at the marginal cost of an extra mass matrix assembly plus a right hand side update (both operations are attributed to elementary terms related to the dual mesh). Concerning the coupling, at the discrete level we introduce a method that follows a monolithic approach, as we aim at constructing solvers that deal with fully coupled systems and employ an exact Jacobian evaluation.

To demonstrate the validity of the proposed numerical scheme for a wide range of parameters and for several levels of model complexity, we consider a series of test cases, highlighting properties such as conservation of constancy and local mass conservation. 
Outline. The paper is organized as follows. First, we introduce a general Eulerian model based on the conservation of mass and momentum for two phases (Section 2). Next, we provide several examples of particular multicontinuum models, which are typically found in the literature and are amenable to the general model ( Section 3). Then, the construction of the proposed mixed FE - primal DFVE scheme applied to the general model is described (Section 4). For each particular scenario, Section 5 collects and discuss some numerical results that demonstrate the validity of the proposed approach and the performance of the hybrid method. We close with some remarks and discussion on possible extensions in Section 6 .

\section{A general multicontinuum model in the context of mixture theory}

Let $T>0$ be a final time and $\Omega \subset \mathbf{R}^{d}$ (with $d=2,3$ the dimension) be an open bounded domain with polyhedral boundary $\Gamma$ and $\nu$ is the outward unit normal vector on $\Gamma$.

\subsection{Balance laws of constituents}

Although an arbitrary number of components can be considered, we restrict the discussion to two components (or phases) $\alpha$ and $\beta$ that occupy the continuum $\Omega$. We denote by $\boldsymbol{u}_{i}, p_{i}, \rho_{i}$, $\mu_{i}$, and $s_{i}$, the velocity, the pressure, the intrinsic material density (assumed to be constant), the viscosity and the volumetric fraction of the phase $i \in\{\alpha, \beta\}$, respectively.

The evolution of the system is described by the mass and momentum conservation equations of the two phases [see, e.g., 2, 6], i.e.,

$$
\begin{aligned}
\partial_{t}\left(s_{i} \rho_{i}\right)+\operatorname{div}\left(s_{i} \rho_{i} \boldsymbol{u}_{i}\right) & =0 \\
\partial_{t}\left(s_{i} \rho_{i} \boldsymbol{u}_{i}\right)+\operatorname{div}\left(s_{i} \rho_{i} \boldsymbol{u}_{i} \otimes \boldsymbol{u}_{i}\right) & =\operatorname{div} \boldsymbol{\sigma}_{i}+\boldsymbol{f}_{i}+s \rho_{i} \boldsymbol{g},
\end{aligned}
$$

where $i \in\{\alpha, \beta\}$ indicates the phase, $\boldsymbol{g}$ is the gravitational acceleration (acting on the negative $x_{d}$-direction), $\boldsymbol{\sigma}_{i}$ and $\boldsymbol{f}_{i}$ are the stress tensors of the phase and the interface momentum exchange terms, respectively, and we have the total volume conservation constraint,

$$
s_{\alpha}+s_{\beta}=1
$$

which expresses the fact that the two phases fill the space. Energy balance equations along with the second law of thermodynamics are here discarded as we restrict the presentation to isothermal mixtures.

To make the system solvable, additional constitutive relationships have to be assigned. For a viscouslinear fluid, for instance, we have

$$
\boldsymbol{\sigma}_{i}:=\mu_{i} \varepsilon\left(\boldsymbol{u}_{i}\right)-\frac{2}{d} \mu_{i}\left(\operatorname{div} \boldsymbol{u}_{i}\right) \mathbf{I}-s_{i} p_{i} \mathbf{I}
$$

where $d$ denotes spatial dimension, I is the identity tensor, and $\varepsilon\left(\boldsymbol{u}_{i}\right)=\frac{1}{2}\left(\nabla \boldsymbol{u}_{i}+\nabla \boldsymbol{u}_{i}^{\mathrm{t}}\right)$ is the infinitesimal rate of strain (for a solid material the elastic shear-stress component has to be added). Setting $s:=s_{\alpha}$, the interface momentum exchange terms are

$$
\boldsymbol{f}_{\alpha}:=-p_{\alpha} \nabla s+D(s)\left(\boldsymbol{u}_{\beta}-\boldsymbol{u}_{\alpha}\right), \quad \boldsymbol{f}_{\beta}:=-p_{\beta} \nabla s+D(s)\left(\boldsymbol{u}_{\alpha}-\boldsymbol{u}_{\beta}\right),
$$

where the first terms represent the effects of the pressure forces (or buoyancy) and the last terms the viscous drag exerted by the other component, which is proportional to the velocity difference and a function of the phase distribution (which is assumed to depend only on the saturation).

Several physical mechanisms are described by an extra energy associated with the interface, which results in a pressure difference between the two phases at equilibrium (e.g., the existence of an interfacial tension between the phases or an effective stress). Here, we follow the notation and the terminology employed in presence of an interfacial tension, and we refer to the pressure difference between the phases as the capillary pressure, $p_{c}$, and assume that it is a function of the saturation

$$
p_{c}(s):=p_{\beta}-p_{\alpha}
$$


The mass and momentum conservation equations for the mixture are obtained by summing up the corresponding equations of the two components. We define the mixture density and the barycentric velocity as

$$
\rho=s \rho_{\alpha}+(1-s) \rho_{\beta} \quad \text { and } \quad \rho \boldsymbol{u}=s \rho_{\alpha} \boldsymbol{u}_{\alpha}+(1-s) \rho_{\beta} \boldsymbol{u}_{\beta},
$$

respectively. Then, we rewrite system (2.1) as

$$
\begin{aligned}
\partial_{t} s+\operatorname{div}\left(s \boldsymbol{u}_{\alpha}\right) & =0 \\
\partial_{t}\left(s \boldsymbol{u}_{\alpha}\right)+\operatorname{div}\left(s \boldsymbol{u}_{\alpha} \otimes \boldsymbol{u}_{\alpha}\right) & =\frac{1}{\rho_{\alpha}} \operatorname{div} \boldsymbol{\sigma}_{\alpha}+\frac{1}{\rho_{\alpha}} \boldsymbol{f}_{\alpha}+s \boldsymbol{g}, \\
\partial_{t} \rho+\operatorname{div}(\rho \boldsymbol{u}) & =0 \\
\partial_{t}(\rho \boldsymbol{u})+\operatorname{div}(\rho \boldsymbol{u} \otimes \boldsymbol{u}) & =\operatorname{div}(\boldsymbol{\sigma}+\boldsymbol{r})-p_{c} \nabla s+\rho \boldsymbol{g},
\end{aligned}
$$

for $(\boldsymbol{x}, t) \in \Omega \times(0, T)$, where $\boldsymbol{r}=-\frac{s \rho_{\alpha}}{(1-s) \rho_{\beta}} \rho\left(\boldsymbol{u}_{\alpha}-\boldsymbol{u}\right) \otimes\left(\boldsymbol{u}_{\alpha}-\boldsymbol{u}\right)$ is the Reynolds stress tensor (which will be neglected from now on), and $\boldsymbol{\sigma}=\boldsymbol{\sigma}_{\alpha}+\boldsymbol{\sigma}_{\beta}$ the total Cauchy stress tensor of the mixture. A constitutive relationship can be directly assigned for the total stress tensor, e.g.,

$$
\boldsymbol{\sigma}=\mu \varepsilon(\boldsymbol{u})-\frac{2}{d} \mu(\operatorname{div} \boldsymbol{u}) \mathbf{I}-p \mathbf{I}
$$

where the total viscosity of the mixture is assumed a function of the saturation, $\mu=\mu(s)$, and

$$
p:=s p_{\alpha}+(1-s) p_{\beta}
$$

is the total pressure. We emphasize that the second term on the RHS of 2.6 describes the force exerted by the interface in case of different pressures; it vanishes if $p_{\alpha}=p_{\beta}$, but is non negligible in general. As we can write

$$
p_{\alpha}(p, s):=p-(1-s) p_{c}(s), \quad \rho(s):=s \rho_{\alpha}+(1-s) \rho_{\beta},
$$

the system (2.3)-(2.6) complemented with appropriate constitutive relationships can be solved for the unknowns $\boldsymbol{u}, \boldsymbol{u}_{\alpha}, s$, and $p$; then, the other variables (e.g., $p_{\alpha}$ and $\rho$ ) can be obtained from (2.7). Other configurations of independent variables are certainly possible and may be more effective depending on the specific application.

\subsection{Weak formulation}

Let us multiply the mass and momentum equations for the mixture and for phase $\alpha$ by suitable test functions. After integrating by parts on $\Omega$, we obtain the weak formulation of the problem: for each $0<t<T$, find $\boldsymbol{u}_{\alpha}(t), \boldsymbol{u}(t), p(t), s(t)$ such that (from now on we drop from the notation the explicit time dependence of the unknowns whenever it is clear from the context)

$$
\begin{array}{r}
\int_{\Omega} \partial_{t}\left(s \boldsymbol{u}_{\alpha}\right) \cdot \boldsymbol{v}_{\alpha} \mathrm{d} \boldsymbol{x}+\mathcal{C}^{s}\left(\boldsymbol{u}_{\alpha} ; \boldsymbol{u}_{\alpha}, \boldsymbol{v}_{\alpha}\right)+\mathcal{A}^{\mu_{\alpha} / \rho_{\alpha}}\left(\boldsymbol{u}_{\alpha}, \boldsymbol{v}_{\alpha}\right)-\mathcal{B}_{s}\left(p_{\alpha}, \boldsymbol{v}_{\alpha}\right)+\mathcal{F}_{1}^{s}\left(p_{\alpha}, \boldsymbol{v}_{\alpha}\right)-\mathcal{F}_{2}^{s}\left(\boldsymbol{u}_{\alpha}, \boldsymbol{u}_{\beta}, \boldsymbol{v}_{\alpha}\right)=\mathcal{G}^{s}\left(\boldsymbol{v}_{\alpha}\right) \\
\int_{\Omega} \partial_{t}(\rho \boldsymbol{u}) \cdot \boldsymbol{v} \mathrm{d} \boldsymbol{x}+\mathcal{C}^{\rho}(\boldsymbol{u} ; \boldsymbol{u}, \boldsymbol{v})+\mathcal{A}^{\mu}(\boldsymbol{u}, \boldsymbol{v})-\mathcal{B}_{1}(p, \boldsymbol{v})+\mathcal{F}_{1}^{s}\left(p_{c}, \boldsymbol{v}\right)=\mathcal{G}^{\rho}(\boldsymbol{v})
\end{array}
$$

for all $\boldsymbol{v}_{\alpha}, \boldsymbol{v} \in \mathbf{H}^{1}(\Omega)$, and

$$
\begin{array}{r}
\int_{\Omega}\left(\partial_{t} s\right) \varphi^{\alpha} \mathrm{d} \boldsymbol{x}+\mathcal{M}_{\boldsymbol{u}_{\alpha}}\left(s, \varphi^{\alpha}\right)+\mathcal{B}_{s}\left(\varphi^{\alpha}, \boldsymbol{u}_{\alpha}\right)=0 \\
\int_{\Omega}\left(\partial_{t} \rho\right) \varphi \mathrm{d} \boldsymbol{x}+\mathcal{M}_{\boldsymbol{u}}(\rho, \varphi)+\mathcal{B}_{\rho}(\varphi, \boldsymbol{u})=0
\end{array}
$$

for all $\varphi^{\alpha}, \varphi \in H_{0}^{1}(\Omega)$, where the involved trilinear, bilinear, and linear forms are defined as

$$
\mathcal{A}^{m}(\boldsymbol{u}, \boldsymbol{v}):=\int_{\Omega} m \boldsymbol{\varepsilon}(\boldsymbol{u}): \boldsymbol{\varepsilon}(\boldsymbol{v}) \mathrm{d} \boldsymbol{x}+\int_{\Omega} \frac{2}{d} m \operatorname{div} \boldsymbol{u} \operatorname{div} \boldsymbol{v} \mathrm{d} \boldsymbol{x}, \quad \mathcal{C}^{m}(\boldsymbol{w} ; \boldsymbol{u}, \boldsymbol{v}):=\int_{\Omega} \operatorname{div}(m \boldsymbol{w} \otimes \boldsymbol{u}) \cdot \boldsymbol{v} \mathrm{d} \boldsymbol{x},
$$




$$
\begin{aligned}
& \mathcal{B}_{\rho}(\varphi, \boldsymbol{v}):=\int_{\Omega} \rho \varphi \operatorname{div} \boldsymbol{v} \mathrm{d} \boldsymbol{x}, \quad \mathcal{G}^{m}(\boldsymbol{v}):=\int_{\Omega} m \boldsymbol{g} \cdot \boldsymbol{v} \mathrm{d} \boldsymbol{x}, \quad \mathcal{M}_{\boldsymbol{v}}(\rho, \varphi):=-\int_{\Omega} \rho(\boldsymbol{v} \cdot \nabla \varphi) \mathrm{d} x, \\
& \mathcal{F}_{1}^{m}(p, \boldsymbol{v}):=\int_{\Omega} p \nabla m \cdot \boldsymbol{v} \mathrm{d} \boldsymbol{x}, \quad \mathcal{F}_{2}^{m}(\boldsymbol{u}, \boldsymbol{v}, \boldsymbol{w}):=\int_{\Omega} D(m)(\boldsymbol{u}-\boldsymbol{v}) \cdot \boldsymbol{w} \mathrm{d} \boldsymbol{x} .
\end{aligned}
$$

Owing to the strongly nonlinear coupling and the possibly degenerate terms in 2.3 - -2.6 , even for regularized versions of the problem it is nontrivial to derive energy estimates, and the analysis of solvability and regularity of the solutions to $2.8-2.9$ is beyond the scope of the present paper. To the best of our knowledge these issues have not been addressed for this kind of formulations, and related studies have dealt mainly with simplified models of single or two-phase flow in porous media [3, 1, 8, 18.

\section{Particular models of archetypal problems}

We now turn to the presentation of some particular models that are structurally similar to each other, and that are amenable to the general system 2.3 - -2.6 , upon adequate manipulation of the governing equations. Such a connection may be straightforward in some cases, but a clear presentation is hardly found in the literature. For completeness, the weak formulations will be rewritten to highlight differences in the structure of the coupled system or in the regularity requirements.

\subsection{Dissolution-driven convection in a porous medium}

We start considering fluid flow through an infinitely rigid and homogeneous porous matrix. Then the velocity of the solid phase is zero, say $\boldsymbol{u}_{\beta}$, and the volumetric fraction, $s_{\beta}$, is constant. Under these conditions, only the conservation equations for the fluid phase, $\alpha$, have to be considered, with a momentum exchange term $\boldsymbol{f}_{\alpha}=-D(s) \boldsymbol{u}_{\alpha}$. Assuming an infinitesimal relaxation time and neglecting the effects of the viscous stress tensor with respect to $\boldsymbol{f}_{\alpha}$, the momentum conservation equation reduces to Darcy's law, and the flow is described by the simple system

$$
\begin{aligned}
\operatorname{div} \boldsymbol{u}_{\alpha} & =0, \\
\boldsymbol{u}_{\alpha} & =-\frac{\kappa}{\mu_{\alpha}}\left(\nabla p_{\alpha}-\rho_{\alpha} \boldsymbol{g}\right) .
\end{aligned}
$$

Let us now consider the case in which the density of the fluid is affected by the presence of a solute, i.e., $\rho_{\alpha}=\rho_{\alpha}(c)$ where $c$ is the concentration, and can give rise to density-driven convection. A typical example is the dissolution and transport of carbon dioxide $\left(\mathrm{CO}_{2}\right)$ into a brine aquifer (see e.g. [27]). Invoking the Boussinesq approximation, (3.1) are still valid, but they have to be complemented by an equation for the concentration, which is typically an advection diffusion equation of the form

$$
\phi \partial_{t} c+\operatorname{div}\left(c \boldsymbol{u}_{\alpha}-\tau_{D} \nabla c\right)=0,
$$

where $\phi$ is the porosity and $\tau_{D}$ is the diffusion coefficient.

For the transport problem 3.2 , inflow and outflow boundary conditions are needed, as well as an initial condition for $c$. For a typical $\mathrm{CO}_{2}$ dissolution problem, a fixed $\mathrm{CO}_{2}$ concentration, $c_{\text {in }}$, is usually assigned at the top boundary $\Gamma_{\mathrm{D}}$ (Dirichlet condition), whereas zero-flux is imposed elsewhere, $\left(c \boldsymbol{u}_{\alpha}-\tau_{D} \nabla c\right) \cdot \boldsymbol{n}=0$ on $\Gamma_{\mathrm{N}}=\Gamma \backslash \Gamma_{\mathrm{D}}$. For the velocity in (3.1) one typically imposes an homogeneous Dirichlet pressure on $\Gamma_{\mathrm{D}}$, and slip velocity $\boldsymbol{u} \cdot \boldsymbol{n}=0$ on $\Gamma_{\mathrm{N}}$.

We emphasize that $(3.2)$ is a mass conservation equation for the solute and the full system of equations ((3.1) and (3.2) has again a structure similar to the general model presented in the previous section, if we consider the solution as a mixture of two miscible component and assume that the velocity of the fluid is the barycentric velocity of the solution. The main differences are that the momentum equation of the solution contains an exchange term with a third component, which is the infinitely rigid porous matrix, and that no momentum equation for the solute is solved, because the difference between the velocity of the solution and the velocity of the solute is modeled by the diffusion term. 
Mixed-primal weak formulation. Multiplication by adequate test functions, using the divergence-free condition on $\boldsymbol{u}_{\alpha}$, and integrating by parts over $\Omega$ yields the following weak formulation of $(3.2)$ : for $t>0$ find $c \in H_{\Gamma}^{1}(\Omega)$ such that

$$
\int_{\Omega} \phi \partial_{t} c w \mathrm{~d} \boldsymbol{x}+\mathcal{M}_{\boldsymbol{u}_{\alpha}}(c, w)+\mathcal{N}_{\tau_{D}}(c, w)=\int_{\Gamma_{\mathrm{D}}} c_{\mathrm{in}} \boldsymbol{u}_{\alpha} \cdot \boldsymbol{n} w \mathrm{~d} C-\int_{\Gamma_{\mathrm{N}}} c \boldsymbol{u}_{\alpha} \cdot \boldsymbol{n} w \mathrm{~d} C \quad \forall w \in H^{1}(\Omega),
$$

where

$$
\mathcal{N}_{\tau_{D}}(c, w):=\int_{\Omega} \tau_{D} \nabla c \cdot \nabla w, \quad \forall c, w \in H^{1}(\Omega) .
$$

Similarly, the weak form of 3.1 reads: for $t>0$ find $\left(\boldsymbol{u}_{\alpha}, p_{\alpha}\right) \in \mathbf{H}(\operatorname{div}, \Omega) \times H_{\Gamma_{\mathrm{D}}}^{1}(\Omega)$ such that

$$
\begin{aligned}
\int_{\Omega} \boldsymbol{u}_{\alpha} \cdot \boldsymbol{v} \mathrm{d} \boldsymbol{x}+\mathcal{B}_{\kappa / \mu}\left(p_{\alpha}, \boldsymbol{v}\right)+\int_{\Gamma_{\mathrm{N}}} \frac{\kappa}{\mu} p_{\alpha} \boldsymbol{v} \cdot \boldsymbol{n} \mathrm{d} C & =\int_{\Omega} \frac{\kappa}{\mu} \rho \boldsymbol{g} \cdot \boldsymbol{v} \mathrm{d} \boldsymbol{x} \quad \forall \boldsymbol{v} \in \mathbf{H}(\mathrm{div}, \Omega), \\
\mathcal{B}_{\kappa / \mu}\left(q, \boldsymbol{u}_{\alpha}\right) & =0 \quad \forall q \in H^{1}(\Omega),
\end{aligned}
$$

where $H_{\Gamma}^{1}(\Omega):=\left\{w \in H^{1}(\Omega):\left.w\right|_{\Gamma}=\psi_{\Gamma}\right\}$. Notice that, in contrast to the weak forms introduced in Section 2.2, the absence of viscosity terms in the momentum equation yields velocity fields that are not necessarily in $\mathbf{H}^{1}(\Omega)$, but only in $\mathbf{H}(\operatorname{div}, \Omega)$ (see $(3.4)$ ).

\subsection{Sedimentation and consolidation of suspensions}

Let us focus now on gravity-driven settling of particles in presence of an interstitial viscous fluid, as modeled in [10] (see also [9, 17, 30]). The main assumptions are as follows: the particles are small with respect to the domain size and have the same density, the constituents of the mixture are incompressible, and the suspension is completely flocculated before the sedimentation takes place.

To describe this phenomenon it is convenient to introduce the average (rather than barycentric) velocity of the mixture

$$
\boldsymbol{q}=s \boldsymbol{u}_{\alpha}+(1-s) \boldsymbol{u}_{\beta},
$$

which, owing to incompressibility of the components and to the mass balance of each phase in (2.1), satisfies $\operatorname{div} \boldsymbol{q}=0$. The mass balance of the solid phase can be then recast as

$$
\partial_{t} s+\operatorname{div}\left(s \boldsymbol{q}+s(1-s) \boldsymbol{u}_{r}\right)=0,
$$

where $\boldsymbol{u}_{r}:=\left(\boldsymbol{u}_{\beta}-\boldsymbol{u}_{\alpha}\right)$ is the filtration velocity (or relative solid-fluid velocity), and $s=s_{\alpha}$ is the volumetric fraction of the solid.

The equations for the average and filtration velocities can be obtained combining the momentum equations in (2.1), which yields to the equivalent system of equations

$$
\begin{aligned}
\operatorname{div} \boldsymbol{q} & =0 \\
\partial_{t} s+\operatorname{div}(s \boldsymbol{q}) & =\operatorname{div}\left(s(1-s) \boldsymbol{u}_{r}\right) \\
\partial_{t} \boldsymbol{q}+\boldsymbol{q} \cdot \nabla \boldsymbol{q} & =\operatorname{div}\left(\boldsymbol{\sigma}_{\alpha} / \rho_{\alpha}\right)+\operatorname{div}\left(\boldsymbol{\sigma}_{\beta} / \rho_{\beta}\right)+\boldsymbol{g}+\boldsymbol{f} \\
\partial_{t} \boldsymbol{u}_{r}+\boldsymbol{q} \cdot \nabla \boldsymbol{u}_{r}+\boldsymbol{u}_{r} \cdot \nabla \boldsymbol{q} & =\frac{1}{\rho_{\alpha} s} \operatorname{div} \boldsymbol{\sigma}_{\alpha}-\frac{1}{\rho_{\beta}(1-s)} \operatorname{div} \boldsymbol{\sigma}_{\beta}-\frac{1}{Q(s)} \boldsymbol{f},
\end{aligned}
$$

where the interaction coefficient is $\boldsymbol{f}=\frac{\rho_{\alpha}-\rho_{\beta}}{\rho_{\alpha} \rho_{\beta}} \boldsymbol{f}_{\beta}, Q(s)=\rho^{-1}\left(\rho_{\alpha}-\rho_{\beta}\right) s(1-s)$, and we have neglected quadratic terms in $\boldsymbol{u}_{r}$ assuming that the filtration velocity is small.

The system can be simplified by assuming a Darcy-like equation for the filtration velocity instead of (3.8). In [10, for instance, a dimensional analysis is performed to show that the momentum equation for the filtration velocity can be replaced by the expression

$$
\boldsymbol{u}_{r}=\frac{b(s)}{\rho g s Q(s)}\left[\nabla \sigma_{e}(s)-s\left(\rho_{\alpha}-\rho_{\beta}\right) \boldsymbol{g}\right]
$$


were $b(s):=u_{\infty} s(1-s)^{2}$ is a batch flux density function describing the effect of hindered settling aligned with the direction of the gravity force [9] $u_{\infty}$ is the constant velocity of a free falling particle in an unbounded medium, $\sigma_{e}=-(p-\hat{p})$ is the effective stress (equal to the difference between the total pressure and the pore-pressure, $\hat{p})$, which is assumed isotropic and a function of solid saturation $\sigma_{e}(s)=\sigma_{0}\left((s l)^{m}-1\right)$, with $m, l$ positive parameters.

Finally, (3.8) can be used to eliminate $\boldsymbol{f}$ from $(3.7)$, and inserting $(3.9)$ into 3.6 we obtain the following initial boundary value problem defined in $\Omega \times(0, T)$ :

$$
\begin{aligned}
\operatorname{div} \boldsymbol{q} & =0, \\
\partial_{t} s+\operatorname{div}(s \boldsymbol{q}-b(s) \boldsymbol{g} / g) & =\operatorname{div}(\kappa(s) \nabla s), \\
\partial_{t} \boldsymbol{q}+\boldsymbol{q} \cdot \nabla \boldsymbol{q}-\frac{1}{\rho} \operatorname{div}(\mu(s) \boldsymbol{\varepsilon}(\boldsymbol{q})-p \mathbf{I}) & =Q(s)\left(\partial_{t} \boldsymbol{u}_{r}+\boldsymbol{q} \cdot \nabla \boldsymbol{u}_{r}\right)+Q(s) \boldsymbol{u}_{r} \cdot \nabla \boldsymbol{q}+\boldsymbol{g},
\end{aligned}
$$

where the volume fraction-dependent coefficient

$$
\kappa(s):=\frac{b(s) \frac{d \sigma_{\alpha}}{d s}}{g\left(\rho_{\alpha}-\rho_{\beta}\right) s},
$$

is a nonlinear diffusivity accounting for compressibility of the sediment, and $\mu(s):=(1-s)^{-3}$ is a power law viscosity depending on the volume fraction [9]. The system is supplemented with the boundary conditions

$$
\boldsymbol{q}=\boldsymbol{q}_{G}, \quad(s \boldsymbol{q}-b(s) \boldsymbol{g}-\kappa(s) \nabla s) \cdot \boldsymbol{\nu}=0, \quad \text { on } \Gamma \times(0, T),
$$

and the initial data $\boldsymbol{q}(0)=\boldsymbol{q}_{0}, s(0)=s_{0}$ in $\Omega \times\{0\}$.

The first two terms in the RHS of the momentum equation of 3.10 account for microstructural arrangement of the granular material, and we will keep them in our numerical tests, even if they are usually discarded in classical models of sedimentation (see e.g. [10] and the references therein).

Mixed-primal weak formulation. As in Section 3.1 the resulting transport equation in 3.10 contains a diffusive term. The weak formulation associated to $(3.10)$ is: for $t>0$ find $s \in H_{\Gamma}^{1}(\Omega)$ such that

$$
\int_{\Omega} \phi \partial_{t} s w \mathrm{~d} \boldsymbol{x}+\mathcal{M}_{\boldsymbol{q}}(s, w)+\mathcal{N}_{\kappa(s)}(s, w)=\int_{\Gamma_{\mathrm{in}}} s_{\mathrm{in}} \boldsymbol{q} \cdot \boldsymbol{n} w \mathrm{~d} S-\int_{\Gamma_{\text {out }}} s \boldsymbol{q} \cdot \boldsymbol{n} w \mathrm{~d} S \quad \forall w \in H^{1}(\Omega),
$$

and $(\boldsymbol{q}, p) \in \mathbf{H}(\operatorname{div}, \Omega) \times L^{2}(\Omega)$ such that

$$
\begin{aligned}
\int_{\Omega} \partial_{t} \boldsymbol{q} \cdot \boldsymbol{v} \mathrm{d} \boldsymbol{x}+\mathcal{C}^{1}(\boldsymbol{q} ; \boldsymbol{q}, \boldsymbol{v})+\mathcal{A}^{\mu(s) / \rho}(\boldsymbol{q}, \boldsymbol{v})-\mathcal{B}_{1}(p, \boldsymbol{v})=\mathcal{H}(s, \boldsymbol{q}, \boldsymbol{v}), \\
\mathcal{B}_{1}(q, \boldsymbol{q})=0
\end{aligned}
$$

for all $(\boldsymbol{v}, q) \in \mathbf{H}^{1}(\Omega) \times L^{2}(\Omega)$, where

$$
\mathcal{H}(s, \boldsymbol{w}, \boldsymbol{v}):=\int_{\Omega}\left(Q(s)\left(\partial_{t} \boldsymbol{u}_{r}(s)+\boldsymbol{w} \cdot \nabla \boldsymbol{u}_{r}(s)\right)+Q(s) \boldsymbol{u}_{r}(s) \cdot \nabla \boldsymbol{w}+\boldsymbol{g}\right) \cdot \boldsymbol{v} \mathrm{d} \boldsymbol{x} .
$$

Even if the total pressure depends implicitly on the effective solid stress function, we notice that the weak formulation only requires $L^{2}(\Omega)$-regularity for $p$, whereas we require the averaged velocity to be in $\mathbf{H}^{1}(\Omega)$.

\subsection{Equations of linear poroelasticity}

Another problem which is amenable to the system $2.3-2.6$ is fluid flow in deformable porous media. As in the previous section, the phase $\alpha$ correspond to the solid and $\beta$ to the fluid.

In standard poroelasticity [see, e.g., 13, inertial terms are neglected in the momentum balance balance of the solid phase, which reads

$$
\operatorname{div} \boldsymbol{\sigma}+\rho \boldsymbol{g}=0
$$


where $\rho$ is the bulk density, $\boldsymbol{g}$ the gravity acceleration, and

$$
\boldsymbol{\sigma}=\boldsymbol{\sigma}_{\alpha}-p \mathbf{I}=\lambda \operatorname{div}(\boldsymbol{d}) \mathbf{I}+2 G \varepsilon(\boldsymbol{d})-p \mathbf{I}
$$

is the effective stress tensor for a linear elastic medium 31, with fluid pressure $p$, Lamé's first parameter $\lambda$, shear modulus $G$, and linearized (infinitesimal) strain tensor $\boldsymbol{\varepsilon}(\boldsymbol{d})=\frac{1}{2}\left(\nabla \boldsymbol{d}+\nabla \boldsymbol{d}^{\mathrm{t}}\right.$ ), where $\boldsymbol{d}$ is the solid displacement. As for the sedimentation and consolidation model described in the previous section, the second momentum equation is simply replaced by Darcy's law for the filtration velocity,

$$
\boldsymbol{u}_{\beta}-\boldsymbol{u}_{\alpha}=-(1-s)^{-1} \frac{\kappa}{\mu_{\beta}}\left(\nabla p-\rho_{\beta} \boldsymbol{g}\right),
$$

where $\kappa$ is the permeability, $s$ the volume fraction of the solid phase, and $(1-s)$ the porosity.

Assuming that deformations are small, the total derivative can be approximated by the partial time derivative, which allows the solid velocity to be written as $\boldsymbol{u}_{\alpha}=\partial_{t} \boldsymbol{d}$, and the fluid conservation equation as

$$
\partial_{t}\left(c_{\beta} p+\alpha \operatorname{div} \boldsymbol{d}\right)-\operatorname{div}\left[\frac{\kappa}{\mu_{\beta}}\left(\nabla p-\rho_{\beta} \boldsymbol{g}\right)\right]=b,
$$

where $b$ is a source term, $\alpha$ is the Biot's coefficient, and $c_{\beta}$ the fluid compressibility. As in [35], we have supposed that the total amount of fluid content depends on the fluid pressure multiplied by a specific storage coefficient $c_{\beta}>0$ plus the material volume. The same system serves as model for the Biot thermoelasticity problem [5], where $p$ has to be interpreted as the temperature field.

Summarizing and introducing the effective solid stress tensor as an additional unknown, the mixed formulation reads: find $\boldsymbol{d}, \boldsymbol{\sigma}_{\alpha}, \boldsymbol{z}$ and $p$ such that

$$
\begin{aligned}
-\operatorname{div}\left(\boldsymbol{\sigma}_{\alpha}-p \mathbf{I}\right) & =\rho \boldsymbol{g}, \\
\boldsymbol{\sigma}_{\alpha} & =\lambda(\operatorname{div} \boldsymbol{d}) \mathbf{I}+2 G \boldsymbol{\varepsilon}(\boldsymbol{d}), \\
\partial_{t}\left(c_{\beta} p+\operatorname{div} \boldsymbol{d}\right)+\operatorname{div} \boldsymbol{z} & =b, \\
\mu_{\beta} \kappa^{-1} \boldsymbol{z}+\nabla p & =\rho_{\beta} \boldsymbol{g} .
\end{aligned}
$$

Boundary and initial data depend on the specific scenario, and here we restrict the presentation to the case of slip velocity and zero-fluid-flux everywhere on $\Gamma$, zero displacements on $\Gamma_{\mathrm{D}}$, no-stress on $\Gamma_{\mathrm{N}}$, and $p(0)=p_{0}$ in $\Omega$.

Mixed-primal and mixed-mixed weak formulations. A mixed-primal variational form associated to (3.11) and $\left(3.12\right.$ can be summarized as follows: for $t>0$, find $(\boldsymbol{d}, p, \boldsymbol{z}) \in \mathbf{H}^{1}(\Omega) \times L^{2}(\Omega) \times \mathbf{H}_{0}(\operatorname{div}, \Omega)$ such that

$$
\begin{aligned}
\mathcal{A}_{2 G}^{\lambda}(\boldsymbol{d}, \boldsymbol{v})-\mathcal{B}_{1}(p, \boldsymbol{v}) & =\int_{\Omega} \rho \boldsymbol{g} \cdot \boldsymbol{v} \mathrm{d} \boldsymbol{x}, \\
\int_{\Omega} \partial_{t} p q \mathrm{~d} \boldsymbol{x}+\mathcal{B}_{1}\left(q, \partial_{t} \boldsymbol{d}\right)+\mathcal{B}_{1}(q, \boldsymbol{z}) & =\int_{\Omega} g q \mathrm{~d} \boldsymbol{x}, \\
\int_{\Omega} \mu_{\beta} \kappa^{-1} \boldsymbol{z} \cdot \boldsymbol{w} \mathrm{d} \boldsymbol{x}-\mathcal{B}_{1}(p, \boldsymbol{w}) & =\mathcal{G}^{\rho_{\beta}}(\boldsymbol{w}),
\end{aligned}
$$

for all $(\boldsymbol{v}, q, \boldsymbol{w}) \in \mathbf{H}^{1}(\Omega) \times L^{2}(\Omega) \times \mathbf{H}_{0}(\operatorname{div}, \Omega)$. In addition, a mixed-mixed variational form corresponding to the coupled problem 3.13 reads:

$$
\begin{aligned}
\int_{\Omega} \boldsymbol{\sigma}_{\alpha}: \boldsymbol{\tau} \mathrm{d} \boldsymbol{x}-\int_{\Omega} \lambda \operatorname{tr}\left(\boldsymbol{\sigma}_{\alpha}\right) \operatorname{tr}(\boldsymbol{\tau})-\int_{\Omega} 2 G \boldsymbol{\varepsilon}(\boldsymbol{d}): \boldsymbol{\tau} \mathrm{d} \boldsymbol{x} & =0 \\
-\int_{\Omega} \operatorname{div} \boldsymbol{\sigma}_{\alpha} \cdot \boldsymbol{v} \mathrm{d} \boldsymbol{x} & =\int_{\Omega} \rho \boldsymbol{g} \cdot \boldsymbol{v} \mathrm{d} \boldsymbol{x} \\
\int_{\Omega} \partial_{t} p q \mathrm{~d} \boldsymbol{x}+\mathcal{B}_{1}\left(q, \partial_{t} \boldsymbol{d}\right)+\mathcal{B}_{1}(q, \boldsymbol{z}) & =\int_{\Omega} g q \mathrm{~d} \boldsymbol{x}
\end{aligned}
$$



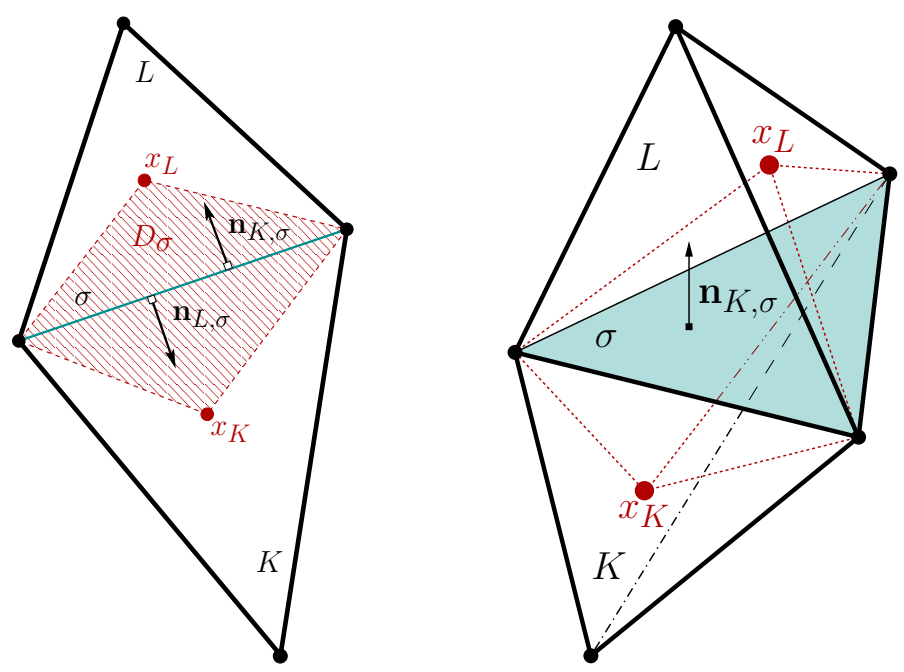

Figure 1: Schematic diagram in two and three-dimensions of two neighbors $K, L$ in the primal mesh $\mathcal{T}_{h}$ (solid lines) sharing the interior face $\sigma$. Their barycenters $\boldsymbol{x}_{K}, \boldsymbol{x}_{L}$ and the vertices of $\sigma$ completely characterize a diamond $D_{\sigma}$ (split into $D_{\sigma}^{K}$ and $D_{\sigma}^{L}$ ) in the dual mesh $\mathcal{T}_{h}^{\sharp}$ (red dashed lines).

$$
\int_{\Omega} \mu_{\beta} \kappa^{-1} \boldsymbol{z} \cdot \boldsymbol{w} \mathrm{d} \boldsymbol{x}-\mathcal{B}_{1}(p, \boldsymbol{w})=\mathcal{G}^{\rho_{\beta}}(\boldsymbol{w})
$$

for all $(\boldsymbol{\tau}, \boldsymbol{v}, q, \boldsymbol{w}) \in \mathbb{H}(\operatorname{div}, \Omega) \times \mathbf{H}^{1}(\Omega) \times L^{2}(\Omega) \times \mathbf{H}_{0}(\operatorname{div}, \Omega)$.

\section{Mixed finite element - primal discontinuous finite-volume-element approximation}

The weak form of the equilibrium and continuity equations derived in Section 2.2 is now adopted to construct a mixed finite element method coupled with a primal DFVE scheme. As previously discussed, the particular models in Section 3 are amenable to the general model and the derivation of their mixed finite element - primal DFVE approximation can be obtain through an analogous procedure.

\subsection{Meshes and preliminaries}

Let $\mathcal{T}_{h}$ be a regular partition of $\Omega$ formed by closed triangular (or tetrahedral in $d=3$ ) elements $K$ with boundary $\partial K$ and diameter $h_{K}$. Each face $\sigma$ between two neighboring elements $K$ and $L$ has diameter $h_{\sigma}$. The set of all faces in $\mathcal{T}_{h}$ is denoted by $\mathcal{E}_{h}$ and $\mathcal{E}_{h}^{\Gamma}$ is its restriction to boundary faces. Associated to the mesh $\mathcal{T}_{h}$ with size $h=\max _{K \in \mathcal{T}_{h}} h_{K}$ we denote by $\mathcal{V}_{h}, \mathcal{Q}_{h}, \mathcal{S}_{h}$ the FE spaces for the approximation of velocity, pressure and a scalar quantity, respectively. Let $\boldsymbol{n}_{K, \sigma}$ denote the outward vector of $K \in \mathcal{T}_{h}$ normal to $\sigma \subset \partial K$. For a scalar function $q \in L^{2}(\Omega)$ we let $\llbracket q \rrbracket_{\sigma}:=\left.q\right|_{\partial K} \boldsymbol{n}_{K, \sigma}+\left.q\right|_{\partial L} \boldsymbol{n}_{L, \sigma}$ denote a vector jump across the face $\sigma=\bar{K} \cap \bar{L}$ and $\{q\}_{\sigma}$ denotes its average value on $\sigma$. For a vectorial function $\boldsymbol{w} \in \mathbf{L}^{2}(\Omega)$ these operators are defined as a tensor-valued jump $\llbracket \boldsymbol{w} \rrbracket_{\sigma}:=\left.\boldsymbol{w}\right|_{\partial K} \otimes \boldsymbol{n}_{K, \sigma}+\left.\boldsymbol{w}\right|_{\partial L} \otimes \boldsymbol{n}_{L, \sigma}$ and $\{\boldsymbol{w}\}_{\sigma}:=\frac{1}{2}\left(\left.\boldsymbol{w}\right|_{\partial K}+\left.\boldsymbol{w}\right|_{\partial L}\right)$. If $\sigma \in \mathcal{E}_{h}^{\Gamma}$ then we consider the convention $\llbracket q \rrbracket_{\sigma}=\{q\}_{\sigma}=\left.q\right|_{\sigma}$ and $\llbracket \boldsymbol{w} \rrbracket_{\sigma}=\boldsymbol{w} \cdot \boldsymbol{\nu}$.

Next, for a given primal mesh $\mathcal{T}_{h}$, a diamond mesh $\mathcal{T}_{h}^{\sharp}$ is introduced, where each diamond $D_{\sigma} \in \mathcal{T}_{h}^{\sharp}$ is associated to the primal face $\sigma \in \mathcal{E}_{h}$ and constructed by joining the barycenters of the primal elements $K$ and $L$ sharing the interior face $\sigma$, with the vertices of $\sigma$ (see Figure 1 for a sketch of the $d=2$ and $d=3$ cases). Each diamond is then intersected with the primal element to generate two sub-diamonds $D_{\sigma}^{K}:=D_{\sigma} \cap K$ and $D_{\sigma}^{L}=D_{\sigma} \cap L$, that conform the control volumes of the diamond mesh. The transfer between meshes represents a projection of the FE space for the approximation of a scalar unknown on the following finite dimensional space

$$
\mathcal{S}_{h}^{\sharp}:=\left\{\phi \in L^{2}(\Omega):\left.\phi\right|_{D_{\sigma}^{K}} \in \mathbb{P}_{0}\left(D_{\sigma}^{K}\right) \forall D_{\sigma}^{K} \in \mathcal{T}_{h}^{\sharp}\right\},
$$


under the action of a projection map $\mathcal{P}^{\sharp}$ characterized by

$$
\left.\mathcal{P}^{\sharp} s_{h}\right|_{D_{\sigma}^{K}}=\frac{1}{h_{\sigma}} \int_{\sigma}\left\{s_{h}\right\}_{\sigma} \mathrm{d} S \text { for all } D_{\sigma}^{K} \in \mathcal{T}_{h}^{\sharp},
$$

where $s_{h} \in \mathcal{S}_{h}$ (see [9]). This operator connects the trial and test spaces, and it is self-adjoint with respect to the $L^{2}$-inner product. Moreover the following result can be established (see [9, 25, 26] for a proof).

Lemma 4.1. For every $s_{h}, \varphi_{h} \in \mathcal{S}_{h}$ and $\boldsymbol{u}_{h} \in \mathcal{V}_{h}$ it holds that

$$
\begin{aligned}
\widehat{\mathcal{N}}\left(s_{h}, \varphi_{h}\right) & :=-\sum_{D_{\sigma}^{K} \in \mathcal{T}_{h}^{\sharp}} \int_{\partial D_{\sigma}^{K}} \kappa\left(s_{h}\right) \nabla s_{h} \cdot \boldsymbol{\nu} \mathcal{P}^{\sharp} \varphi_{h} \mathrm{~d} S=\mathcal{N}_{\kappa}\left(s_{h}, \varphi_{h}\right)-\sum_{\sigma \in \mathcal{E}_{h}} \int_{\sigma}\left\{\kappa\left(s_{h}\right) \nabla s_{h}\right\} \llbracket \mathcal{P}^{\sharp} \varphi_{h} \rrbracket \mathrm{d} S, \\
\widehat{\mathcal{M}}_{\boldsymbol{u}_{h}}\left(s_{h}, \varphi_{h}\right) & :=\sum_{D_{\sigma}^{K} \in \mathcal{T}_{h}^{\sharp}} \int_{\partial D_{\sigma}^{K}} s_{h} \boldsymbol{u}_{h} \cdot \boldsymbol{\nu} \mathcal{P}^{\sharp} \varphi_{h} \mathrm{~d} S \\
& =\mathcal{M}_{\boldsymbol{u}_{h}}\left(s_{h}, \varphi_{h}\right)+\mathcal{B}_{s_{h}}\left(\varphi, \boldsymbol{u}_{h}\right)-\sum_{\sigma \in \mathcal{E}_{h}} \int_{\sigma}\left\{s_{h} \boldsymbol{u}_{h} \cdot \boldsymbol{\nu}\right\} \llbracket \mathcal{P}^{\sharp} \varphi_{h} \rrbracket \mathrm{d} S,
\end{aligned}
$$

where $\mathcal{M} .(\cdot, \cdot), \mathcal{B} .(\cdot, \cdot)$ are defined as in $(2.9)$, and $\mathcal{N}(\cdot, \cdot)$ as in 3.3$)$.

\subsection{Statement of the combined mixed-primal scheme}

Let us assume that the discrete space of the approximation of the velocity (phase or total velocity), $\mathcal{V}_{h}$, is a subspace of $\mathbf{H}^{1}(\Omega)$, and let us consider the following semidiscrete, nonlinear Galerkin scheme associated to (2.8). For a given $t>0$, find $\boldsymbol{u}_{h}^{\alpha}, \boldsymbol{u}_{h} \in \mathcal{V}_{h}$ such that

$$
\begin{gathered}
\sum_{K \in \mathcal{T}_{h}} \int_{K} \partial_{t} s_{h} \boldsymbol{u}_{h}^{\alpha} \cdot \boldsymbol{v}_{h}^{\alpha} \mathrm{d} \boldsymbol{x}+\mathcal{C}^{s_{h}}\left(\boldsymbol{u}_{h}^{\alpha} ; \boldsymbol{u}_{h}^{\alpha}, \boldsymbol{v}_{h}^{\alpha}\right)+\mathcal{A}^{\mu_{\alpha} / \rho_{\alpha}}\left(\boldsymbol{u}_{h}^{\alpha}, \boldsymbol{v}_{h}^{\alpha}\right)-\mathcal{B}_{s_{h}}\left(p_{h}^{\alpha}, \boldsymbol{v}_{h}^{\alpha}\right) \\
+\mathcal{F}_{1}^{s_{h}}\left(p_{h}^{\alpha}, \boldsymbol{v}_{h}^{\alpha}\right)-\mathcal{F}_{2}^{s_{h}}\left(\boldsymbol{u}_{h}^{\beta}, \boldsymbol{v}_{h}^{\alpha}\right)=\mathcal{G}^{s_{h}}\left(\boldsymbol{v}_{h}^{\alpha}\right), \\
\sum_{K \in \mathcal{T}_{h}} \int_{K} \partial_{t} \rho_{h} \boldsymbol{u}_{h} \cdot \boldsymbol{v}_{h} \mathrm{~d} \boldsymbol{x}+\mathcal{C}^{\rho_{h}}\left(\boldsymbol{u}_{h} ; \boldsymbol{u}_{h}, \boldsymbol{v}_{h}\right)+\mathcal{A}^{\mu\left(s_{h}\right)}\left(\boldsymbol{u}_{h}, \boldsymbol{v}_{h}\right)-\mathcal{B}_{1}\left(p_{h}, \boldsymbol{v}_{h}\right)+\mathcal{F}_{1}^{s_{h}}\left(p_{c, h}, \boldsymbol{v}_{h}\right)=\mathcal{G}^{\rho}\left(\boldsymbol{v}_{h}\right),
\end{gathered}
$$

for all $\boldsymbol{v}_{h}^{\alpha}, \boldsymbol{v}_{h} \in \mathcal{V}_{h}$. In 4.2 it is implicitly assumed that $\mathcal{V}_{h}$ is a subspace of $\mathbf{H}_{0}^{1}(\Omega)$; otherwise, some extra terms will arise after integration by parts, which are typically penalized (see e.g. [32]).

Multiplying the transport equations (2.3) and 2.5) by $\mathcal{P}^{\sharp} \varphi_{h}^{\alpha} \in \mathcal{S}_{h}^{\sharp}$ and $\mathcal{P}^{\sharp} \varphi_{h} \in \mathcal{S}_{h}^{\sharp}$, respectively, and integrating by parts over each diamond $D_{\sigma}^{K} \in \mathcal{T}_{h}^{\sharp}$, we obtain a continuous-time pure FV formulation for the transport problems, since all quantities can be written on the faces of each diamond:

$$
\begin{gathered}
\int_{\Omega} \partial_{t} s_{h} \mathcal{P}^{\sharp} \varphi_{h}^{\alpha} \mathrm{d} \boldsymbol{x}+\sum_{D_{\sigma}^{K} \in \mathcal{T}_{h}^{\sharp}} \int_{\partial D_{\sigma}^{K}} s_{h} \boldsymbol{u}_{h} \cdot \boldsymbol{\nu} \mathcal{P}^{\sharp} \varphi_{h}^{\alpha} \mathrm{d} S=0, \\
\int_{\Omega} \partial_{t} \rho_{h} \mathcal{P}^{\sharp} \varphi_{h} \mathrm{~d} \boldsymbol{x}+\sum_{D_{\sigma}^{K} \in \mathcal{T}_{h}^{\sharp}} \int_{\partial D_{\sigma}^{K}} \rho_{h} \boldsymbol{u}_{h} \cdot \boldsymbol{\nu} \mathcal{P}^{\sharp} \varphi_{h} \mathrm{~d} S=0,
\end{gathered}
$$

for all $\mathcal{P}^{\sharp} \varphi_{h}^{\alpha}, \mathcal{P}^{\sharp} \varphi_{h} \in \mathcal{S}_{h}^{\sharp}$. The DFVE formulation implies the presence of discontinuous fluxes across edges of the primal mesh. We therefore apply Lemma 4.1 along with a generalized upwinding procedure (see e.g. [16]), leading to the scheme

$$
\begin{array}{r}
\int_{\Omega} \partial_{t} s_{h} \mathcal{P}^{\sharp} \varphi_{h}^{\alpha} \mathrm{d} \boldsymbol{x}+\widetilde{\mathcal{M}}_{\boldsymbol{u}_{\alpha, h}}\left(s_{h}, \varphi_{h}^{\alpha}\right)=0, \\
\int_{\Omega} \partial_{t} \rho_{h} \mathcal{P}^{\sharp} \varphi_{h} \mathrm{~d} \boldsymbol{x}+\widetilde{\mathcal{M}}_{\boldsymbol{u}_{h}}\left(\rho_{h}, \varphi_{h}\right)=0,
\end{array}
$$


with

$$
\widetilde{\mathcal{M}}_{\boldsymbol{v}_{h}}\left(s_{h}, \varphi_{h}\right):=\widehat{\mathcal{M}}_{\boldsymbol{v}_{h}}\left(s_{h}, \varphi_{h}\right)+\sum_{\sigma \in \mathcal{E}_{h}} \frac{1}{2} \int_{\sigma}\left|\boldsymbol{v}_{h} \cdot \boldsymbol{\nu}\right| \llbracket s_{h} \rrbracket \cdot \boldsymbol{\nu} \mathcal{P}^{\sharp} \varphi_{h} \mathrm{~d} S,
$$

for all $s_{h}, \varphi_{h}, \boldsymbol{v}_{h}$. In summary, 4.4 contains an edge average-jump term resulting from integration by parts, flux upwinding, and a mass term involving the dual mesh. The complete semidiscrete hybrid mixed FE - primal DFVE formulation is then given by combining 4.4 and 4.2.

The following remarks are noteworthy:

- The discrete formulations given above are valid for any set of finite dimensional spaces such that $\mathcal{V}_{h} \subset \mathbf{H}_{0}^{1}(\Omega)$ (conforming approximation or nonconforming finite element space $\mathcal{V}_{h} \subset \mathbf{H}(\operatorname{div}, \Omega)$ ), $\mathcal{Q}_{h} \subset L^{2}(\Omega)$, and $\mathcal{S}_{h} \subset H^{1}(\Omega)$. Here, however, we will restrict ourselves to the following specific finite dimensional spaces

$$
\begin{aligned}
& \mathcal{V}_{h}=\left\{\boldsymbol{v}:\left.\boldsymbol{v}\right|_{K} \in \operatorname{BDM}_{1}(K), \forall K \in \mathcal{T}_{h}\right\}, \quad \mathcal{Q}_{h}=\left\{q:\left.q\right|_{K} \in \mathbb{P}_{0}(K), \forall K \in \mathcal{T}_{h}\right\}, \\
& \mathcal{S}_{h}=\left\{\phi \in H_{0}^{1}(\Omega):\left.\phi\right|_{K} \in \mathbb{P}_{1}(K), \forall K \in \mathcal{T}_{h}\right\}, \quad \mathcal{S}_{h}^{\sharp}=\left\{\phi \in L^{2}(\Omega):\left.\phi\right|_{D_{\sigma}^{K}} \in \mathbb{P}_{0}\left(D_{\sigma}^{K}\right) \forall D_{\sigma}^{K} \in \mathcal{T}_{h}^{\sharp}\right\},
\end{aligned}
$$

where the local Brezzi-Douglas-Marini spaces of order $k, k \geq 0$ are $\mathbf{B D M}_{k}(K)=\mathbb{P}_{k}(K)^{d} \cap \mathbf{H}(\operatorname{div}, K)$, for $\boldsymbol{x} \in \mathbf{R}^{d}$ (see e.g. [20]).

This approximation of velocities is non-conforming and requires a stabilization term in the momentum and mass equations. We adopt the one proposed in [23] for Brinkman flows.

- After rewriting (4.3) as in 4.4, only the mass terms (temporal derivatives) and the nonzero right hand side involve quantities lying on the dual mesh $\mathcal{T}_{h}^{\sharp}$. These matrices are assembled only at the beginning of the computation, which implies that the projection map between the primal and dual mesh defined in (4.1) is invoked only once, and therefore the overhead computational burden of a dual mesh is negligible.

- Using an extension of the operator defined in 4.1), it is possible to construct a full DFVE scheme for the discretization of 2.3)-2.6). This simply requires replacing 4.2 by a DFVE formulation of velocity and pressure as proposed for instance in [14, 26, for Stokes-related problems. This can be done using either stabilized discontinuous Galerkin $\mathbb{P}_{1}-\mathbb{P}_{1}$ approximations of velocity and pressure, or local pressure projection stabilizations. Such a method would be appealing essentially because of its unified structure. On the other hand, the main advantage of the present mixed-primal global scheme, is that no stabilization parameters are needed in the flow approximation (i.e. of velocity and pressure) in order to comply with the discrete inf-sup condition.

- Discrete consistency with continuity for conforming FVE methods can be proven by adding artificial diffusion to the transport problems, uncoupling them from the momentum equations, and applying monotonicity and Lipschitz continuity assumptions on the total flux (see [19]). In contrast, for the DFVE method, a rigorous proof of discrete maximum principles 4.4 remains an open problem. However, it is still possible to verify this property by means of numerical experiments, as it will be done later in Section 5.1.

- Notice that for Darcy-based models, as the one outlined in Section 3.1, one could also consider lowest order Raviart-Thomas elements $\left(\mathbf{R T}_{k}(K)=\mathbb{P}_{k}(K)^{d} \oplus \mathbb{P}_{k}(K) \boldsymbol{x}\right.$, for $\left.\boldsymbol{x} \in \mathbf{R}^{d}\right)$, requiring only $\mathbf{H}(\operatorname{div}, \Omega)$ regularity for the velocity (since the viscous term does not appear in the momentum equation), which are still compatible with piecewise-constant pressure approximations.

\subsection{Time discretization}

Let $s_{h}^{n+1}$ denote the approximation (either via mixed FE or DFVE) of a generic scalar or vector function $s$ at the time level $t^{n+1}=(n+1) \Delta t, n=0, \ldots, T / \Delta t$, where $\Delta t$ denotes a constant time step. After the spatial discretization of the model problem discussed above, we obtain a large system of nonlinear ODEs 

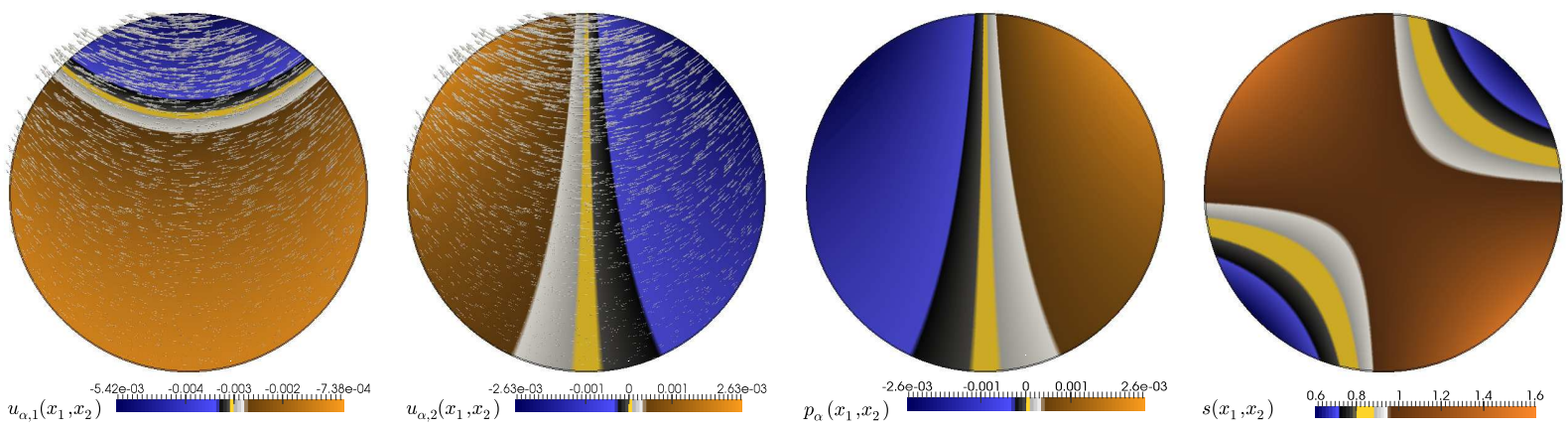

Figure 2: Example 1: snapshots of the approximate velocity components, phasial pressure distribution, and saturation profiles computed at $t=0.2$ for a two-phase flow accuracy test.

4.2)- 4.4 which is integrated in time using the second order backward difference formula (BDF2), i.e., all time derivatives are approximated using the centered operator

$$
\partial_{t} s_{h}\left(t^{n+1}\right) \approx \frac{1}{\Delta t}\left(\frac{3}{2} s_{h}^{n+1}-2 s_{h}^{n}+\frac{1}{2} s_{h}^{n-1}\right),
$$

and for the first time step a first order backward Euler method is used from $t^{0}$ to $t^{1}$. Convective and reactive nonlinearities along with the implicit part of the time integration require a linearization strategy. Here we opt for an iterative Newton-Raphson method involving the full Jacobian matrix of the system. Adopting a formulation in terms of phase velocity, total velocity, saturation and density, the final set of linear equations to be solved at each Newton step $k$, can be represented in matrix form as

$$
\left(\begin{array}{cccc}
\mathbb{A}_{1} & \mathbb{B}_{1} & \mathbf{0} & \mathbf{0} \\
\mathbb{D} & \mathbb{C}_{1} & \mathbf{0} & \mathbf{0} \\
\mathbf{0} & \mathbb{E} & \mathbb{A}_{2} & \mathbb{B}_{2} \\
\mathbf{0} & \mathbf{0} & \mathbb{D} & \mathbb{C}_{2}
\end{array}\right)\left(\begin{array}{c}
\delta \boldsymbol{u}_{\alpha, h}^{k+1} \\
\delta s_{h}^{k+1} \\
\delta \boldsymbol{u}_{h}^{k+1} \\
\delta \rho_{h}^{k+1}
\end{array}\right)=\mathbb{R H} \mathbb{S},
$$

where the $\delta(\cdot)^{k+1}$ stand for the sought incremental solutions, and $\mathbb{R} H \mathbb{S}$ contains all contributions from previous time steps, forcing terms, and Newton residuals. For instance, matrix $\mathbb{A}_{2}$ arises from assembly of the (semi-discrete) terms $M_{2}+C_{2}+C_{3}+A_{1}$ :

$$
\left(\partial_{t} \delta \boldsymbol{u}_{h}^{k+1} \rho_{h}^{k}, \boldsymbol{v}_{h}\right)_{\Omega}+\left(\operatorname{div}\left(\rho_{h}^{k} \delta \boldsymbol{u}_{h}^{k+1} \otimes \boldsymbol{u}_{h}^{k}\right), \boldsymbol{v}_{h}\right)_{\Omega}+\left(\operatorname{div}\left(\rho_{h}^{k} \boldsymbol{u}_{h}^{k} \otimes \delta \boldsymbol{u}_{h}^{k+1}\right), \boldsymbol{v}_{h}\right)_{\Omega}+\left(\mu\left(s_{h}^{k}\right) \boldsymbol{\varepsilon}\left(\delta \boldsymbol{u}_{h}^{k+1}\right), \boldsymbol{\varepsilon}\left(\boldsymbol{v}_{h}\right)\right)_{\Omega}
$$

whereas, making abuse of notation, the remaining blocks are $\mathbb{A}_{1}=M_{2}+C_{2}+C_{3}+A_{1}, \mathbb{B}_{1}=$ $M_{1}+C_{1}+B_{1}+B_{2}+F_{11}+F_{12}+F_{21}+F_{22}-M_{3}, \mathbb{D}=D_{2}+B_{2}^{T}, \mathbb{C}_{1}=N_{1}+D_{1}+B_{1}^{T}, \mathbb{E}=A_{2}+B_{1}+B_{2}+F_{1}$, $\mathbb{B}_{2}=M_{1}+C_{1}-M_{3}$, and $\mathbb{C}_{2}=N_{1}+D_{1}+B_{1}^{T}+F_{2}$.

\section{Numerical results}

In this section we illustrate the behavior of the proposed mixed-primal numerical method by means of several numerical test cases. Unless otherwise specified, we adopt first order Brezzi-Douglas-Marini approximations for velocities, piecewise constant FE for pressures, and DFVE approximations for all remaining scalar fields. Incremental Newton iterations with a fixed tolerance of $10^{-7}$ for the energy norm of the residuals are applied to linearize the algebraic equations arising from the discrete scheme (4.2)-(4.4). Triangular and tetrahedral primal and diamond grids were generated with the open source mesh manipulator Gmsh 22. Large linear systems arising from the Newton linearization algorithm have been solved with the generalized minimum residual (GMRES) method employing an incomplete LU preconditioner without fill-in and a residual tolerance of $10^{-8}$, whereas small systems (typically associated to $2-\mathrm{D}$ and single phase simulations with less than 1M degrees of freedom) were solved with the multifrontal massively parallel sparse direct solver (MUMPS). Multi-thread computations have been carried out on an Intel based cluster with eight dual Xeon nodes of 12 cores, $2.97 \mathrm{GHz}$ CPU and 96 GB RAM, using Infiniband $40 \mathrm{~Gb} / \mathrm{s}$ interconnectivity. 


\begin{tabular}{|c|c|c|}
\hline Example & 5.1 & $r=1, \phi=\kappa=\mu=\rho=1, \tau_{D}=0.01, T=0.2, \Delta t \in[1 / 1280,1 / 10]$ \\
\hline Example & 5.2 & $\begin{array}{l}W_{A}=2000, W_{B}=1, \mathrm{Ra}_{A}=1, \mathrm{Ra}_{B}=10000, p_{0}=\boldsymbol{u}_{0}=0 \\
T_{A}=80000, T_{B}=5, \Delta t_{A}=40, \Delta t_{B}=0.0025\end{array}$ \\
\hline Example & 5.3 & $\begin{array}{l}W=0.1, H=15, K=1.82 \times 10^{-10}, \phi=0.375, G=10^{-6}, p_{0}=0 \\
p_{\partial}=10000, c_{s}=3.48 \times 10^{-5}, c_{f}=1.25 \times 10^{-5}, T=1000, \Delta t=0.1, c_{\beta}=0.01\end{array}$ \\
\hline Example & 5.4 & $\begin{array}{l}W=1, \gamma=0.05, K=10^{-7}, E=10000, \nu=0.4, p_{0}=\boldsymbol{u}_{0}=0, c_{\beta}=10^{-5} \\
\lambda=14285.7142, G=3571.4285, T=50, \Delta t=1, p_{\partial}=20\end{array}$ \\
\hline Example & 5.5 & $W=3, H=25.6, p_{0}=\boldsymbol{u}_{0}=0, \theta=\pi / 3, T=20000, h=2.5, \Delta t=0.01$ \\
\hline Example & 5.6 & $W=1, a=-2.5, \rho_{\beta}=2200, \rho_{\alpha}=1000, m=2, b=0.01, \mu_{\alpha}=\mu_{\beta}=1$ \\
\hline Example & 5.7 & $\begin{array}{l}R=1, H=8, \theta=\pi / 4, \rho_{\alpha}=2640, j=0.0005, \rho_{\beta}^{A}=1000, \mu_{\alpha}=10^{-6}, \mu_{\beta}^{A}=0.001 \\
\rho_{\beta}^{B}=1.225, \mu_{\beta}^{B}=1.7894 \times 10^{-5}, s_{0}^{A}=0.44, s_{0}^{B}=0.15, s_{\max }=0.59, q_{\beta}=\pi / 5\end{array}$ \\
\hline
\end{tabular}

Table 1: Modeling and discretization adimensional parameters adopted for all numerical tests.
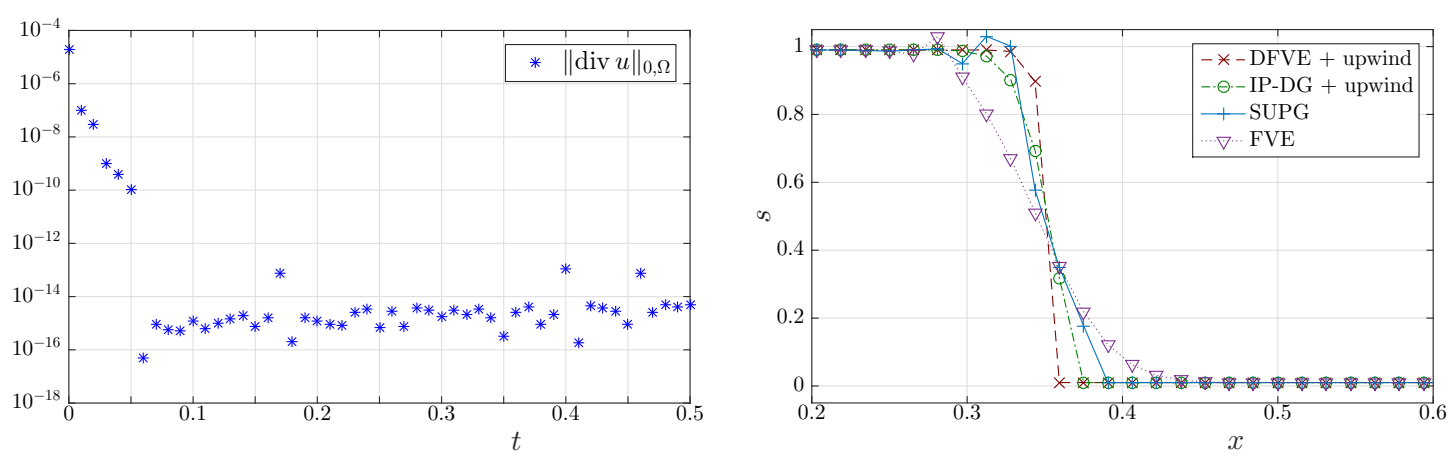

Figure 3: Example 1: evolution of the divergence of total velocity for an homogeneous flow (right), and snapshot of a saturation front captured with different discretizations for the mass conservation equation(right).

\subsection{Example 1: spatio-temporal accuracy and mass conservation}

We start by assessing the accuracy of the numerical scheme. We consider a 2-D domain partitioned into primal unstructured triangulations with $3 \cdot 2^{n+1}(n=0,1, \ldots, 7)$ vertices on the domain boundary. The test case corresponds to the flow of a single-phase in a porous medium and it is governed by 3.2)-3.1). The problem is defined on a disk of radius $r$ and on the time interval $(0, T)$, with $\Gamma_{D}=\Gamma$. We choose parameters and forcing terms so that the exact solutions of the problem are given by the smooth functions

$$
\boldsymbol{u}_{\alpha}(\boldsymbol{x}, t)=\left(\begin{array}{l}
-\cos \left(x_{1}\right) \exp \left(x_{2}\right) \sin (t) \\
-\sin \left(x_{1}\right) \exp \left(x_{2}\right) \sin (t)
\end{array}\right), p_{\alpha}(\boldsymbol{x}, t)=\sin \left(x_{1}\right) \exp \left(x_{2}\right) \sin (t), s(\boldsymbol{x}, t)=\exp \left(-x_{1} x_{2}\right) \cos (t)
$$

In this case, the right hand side of the first equation in 3.2$)$ is

$$
f=-\exp \left(-x_{1} x_{2}\right) \sin (t)+\exp \left(x_{2}\left(1-x_{1}\right)\right) \sin (t) \cos (t)\left[x_{2} \cos \left(x_{1}\right)+x_{1} \sin \left(x_{2}\right)\right]-\tau_{D} \cos (t) \exp \left(-x_{1} x_{2}\right)\left[x_{1}^{2}+x_{2}^{2}\right] \text {. }
$$

We study the accuracy of the discretization using piecewise constant approximations for the pressure, $\mathbf{B D M}_{1}$ approximations for velocities, and discontinuous FVE approximations for saturation. Convergence histories are obtained by computing errors in the natural norms and the convergence rates at a fixed time 


\begin{tabular}{|c|c|c|c|c|c|c|}
\hline \multicolumn{7}{|c|}{ Spatial convergence } \\
\hline$h$ & $\mathrm{e}_{1}(s)$ & $r_{1}(s)$ & $\mathrm{e}_{\operatorname{div}}(\boldsymbol{u})$ & $r_{\text {div }}(\boldsymbol{u})$ & $\mathrm{e}_{0}(p)$ & $r_{0}(p)$ \\
\hline 0.9321 & 0.6221 & - & 0.0042 & - & 0.0041 & - \\
\hline 0.8212 & 0.5237 & 1.0239 & 0.0023 & 0.7943 & 0.0015 & 0.9163 \\
\hline 0.3817 & 0.2411 & 0.7779 & 0.0012 & 0.8964 & 0.0008 & 1.0212 \\
\hline 0.2032 & 0.1187 & 1.0547 & 0.0007 & 0.9134 & 0.0004 & 0.9344 \\
\hline 0.1095 & 0.0596 & 1.1143 & $3.49 \mathrm{e}-4$ & 0.9074 & 0.0002 & 0.9494 \\
\hline 0.0531 & 0.0304 & 0.9722 & $1.85 \mathrm{e}-4$ & 0.9671 & 0.0001 & 0.9107 \\
\hline 0.0309 & 0.0150 & 1.0347 & $7.96 \mathrm{e}-5$ & 1.0542 & $6.14 \mathrm{e}-5$ & 1.1031 \\
\hline 0.0147 & 0.0076 & 0.9821 & $4.41 \mathrm{e}-5$ & 0.9569 & $2.93 e-5$ & 0.9856 \\
\hline \multicolumn{7}{|c|}{ Temporal convergence } \\
\hline$\Delta t$ & $\mathrm{E}_{\ell}^{1}(s)$ & $R_{\ell}^{1}(s)$ & $\mathrm{E}_{\ell}^{\operatorname{div}}(\boldsymbol{u})$ & $R_{\ell}^{\text {div }}(\boldsymbol{u})$ & $\mathrm{E}_{\ell}^{1}(p)$ & $R_{\ell}^{1}(p)$ \\
\hline 0.1000 & 321.33 & - & 5.0190 & - & 0.2955 & - \\
\hline 0.0500 & 85.385 & 2.0321 & 1.1344 & 1.9215 & 0.0640 & 1.8897 \\
\hline 0.0250 & 28.324 & 1.9842 & 0.2831 & 1.9350 & 0.0165 & 1.9713 \\
\hline 0.0125 & 7.1521 & 1.8853 & 0.6399 & 1.8818 & 0.0044 & 1.8796 \\
\hline 0.0062 & 1.8344 & 1.8901 & 0.1640 & 1.8923 & 0.0013 & 1.9307 \\
\hline 0.0031 & 4.2230 & 1.9840 & 0.0451 & 1.9543 & 0.0003 & 1.9146 \\
\hline 0.0015 & 1.1391 & 2.0121 & 0.0129 & 1.9522 & 0.0001 & 1.9328 \\
\hline
\end{tabular}

Table 2: Example 1: experimental space and time convergence until $T=0.2$ for the mixed FE - primal DFVE approximation of the single-phase flow problem $3.2,3.1$.

$t^{N}=T$, are defined as

$$
\mathrm{e}_{i}(m):=\left\|m^{N}-m_{h}^{N}\right\|_{i, \Omega}, r_{i}(m):=\frac{\log \left(\mathbf{e}_{i}(m) / \hat{\mathbf{e}}_{i}(m)\right)}{\log (h / \hat{h})},
$$

with $i \in\{\operatorname{div}, 0,1\}$, for any regular function $m^{N}=s(\cdot, T)$ (velocity component, saturation, or pressure), where $e$ and $\hat{e}$ denote errors computed on two consecutive meshes $\mathcal{T}_{h}$ and $\mathcal{T}_{\hat{h}}$, respectively. Temporal accuracy is evaluated by computing errors and rates in the $\ell^{\infty}(0, t ; \cdot)$-norm

$$
\mathrm{E}_{\ell}^{i}(m):=\sum_{n=0}^{N}\left\|m^{n}-m_{h}^{n}\right\|_{i, \Omega}, \quad R_{\ell}^{i}(m):=\frac{\log \left(\mathrm{E}_{\ell}^{i}(m) / \hat{\mathrm{E}}_{\ell}^{i}(m)\right)}{\log (\Delta t / \widehat{\Delta t})},
$$

on successively refined partitions of $(0, T)$, where $i \in\{\operatorname{div}, 0,1\}$.

The convergence behavior is reported in Table 2, where the third, fifth and seventh columns of the table indicate a first order convergence in space and second order convergence in time. Given the present spatial and temporal discretization, these rates are optimal in the sense that errors decay with the same order as the corresponding interpolator operators. Approximate solutions are depicted in Figure 2 and are free from spurious pressure patterns.

Next, we test the ability of the scheme to recover the homogeneous flow solution. We set $s=\rho=1$ and solve the multiphase flow problem using the same parameters as in the example above. Since the phase and total velocities are approximated with $H$ (div)-conforming methods, their normal components are continuous across element boundaries and accurate velocity profiles are expected. The left plot in Figure 3 shows the evolution of the divergence of the total velocity, which stays around 1e-14 for most of the simulation, indicating that incompressible flow is correctly recovered.

Finally, we solve a pseudo-1D transport problem including a regularizing diffusion term:

$$
\partial_{t} s+\partial_{x}(s u)-D \partial_{x x} s=f s,
$$




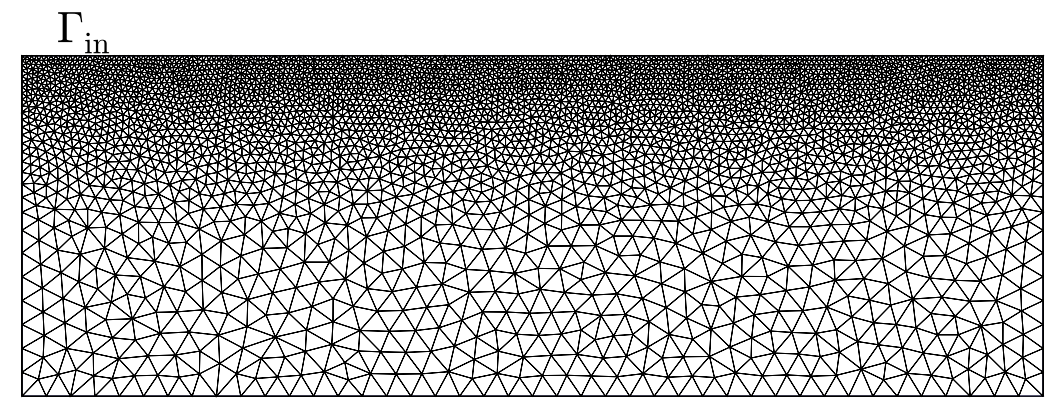

Figure 4: Example 2: zoom of the unstructured primal mesh, where we observe an isotropic refinement near the top of the domain, where the inlet boundary $\Gamma_{\text {in }}$ is located.

defined in $\Omega=(0,2)$ for $t>0$ and with $D=5 e-4$. A coarse mesh of 64 elements is used, and an exact velocity and $\operatorname{sink}$ are considered $u=\cos (\pi x / 2), f=-\pi / 2 \sin (\pi x / 2)$ (see [15]). The initial condition is $s(0)=0.01$ and the inflow saturation (at the left boundary) is $s=0.99$. A fixed time step of $\Delta t=1 e-4$ is used and the system is evolved until $T=0.5$. We compare the results obtained with 4.4 against those generated with the following schemes: a classical interior penalty discontinuous Galerkin method (IP-DG) using the same upwind numerical flux as in 4.4, a (continuous) finite volume element method (FVE, which is locally conservative in the dual mesh), and a classical stream-upwind Petrov-Galerkin stabilized method (SUPG). A snapshot of the solution at an intermediate time is shown in the right panel of Figure 3 (zoomed into the wave front). We observe that regardless of the used method, no undershoot occurs, but a violation of the maximum principle is observed with the FVE and SUPG methods. The continuous FVE method introduces numerical diffusion; the SUPG method is less diffusive, but produces spurious oscillations near the large gradient regions. The IP-DG method exhibits no oscillations, but is more diffusive than the proposed DFVE scheme, which provides the most accurate solution among the numerical scheme that we have tested.

\subsection{Example 2: gravity-driven instabilities}

We now study the gravity-driven instabilities arising in the study of $\mathrm{CO}_{2}$ storage in saline aquifers. We consider a square domain $\Omega=(0, W) \times(-W, 0)$ and we notice that the only free parameter is the Rayleigh number $\mathrm{Ra}=\frac{\kappa \rho}{\phi \tau_{D} \mu}$, if the system is written in dimensionless form. We assume zero initial pressure and velocity, whereas a random concentration value is assigned near the top boundary and zero elsewhere. No artificially selected wavelength is encountered. A free-slip condition is imposed for the velocity everywhere except at the top boundary, where we fixed the pressure. A diffusive influx of carbon dioxide into the brine aquifer is modeled by imposing a constant maximum concentration at the top, whereas zero-flux is assigned at the other boundaries. An unstructured mesh of 237'444 primal triangular elements and 118'723 nodes has been employed for the simulations (for a detail of the mesh see Figure 4). The mesh resolution is adequate to capture the main flow features and no qualitative difference in the fingering patterns is observed when the mesh is refined.

Figures 5 and 6 show the finger growth and the corresponding magnitude of the velocity, which exhibits nonzero flow normal to the inlet boundary. Notice that the finger dynamics (shape and frequency distribution) changes substantially between the two regimes dictated by a different Rayleigh number. The time step and total simulation times have also been rescaled accordingly (see Table 1). In the first case (test A), larger fingers with a strong diffusive spreading are evident, whereas in the second case (test B) we observe secondary instabilities and the formation of lobes of carbon dioxide that start diffusing into the surrounding brine. These results agree with the simulations reported in [27. A more quantitative assessment is displayed in Figure 7. The first row panels show the temporal trend of the number of fingers, the total amount of carbon dioxide in the domain, and the total pressure distribution. The first indicator shows an average of 7 fingers for test A and 16 fingers for test B. As in 34] we also compare the carbon dioxide concentration and 

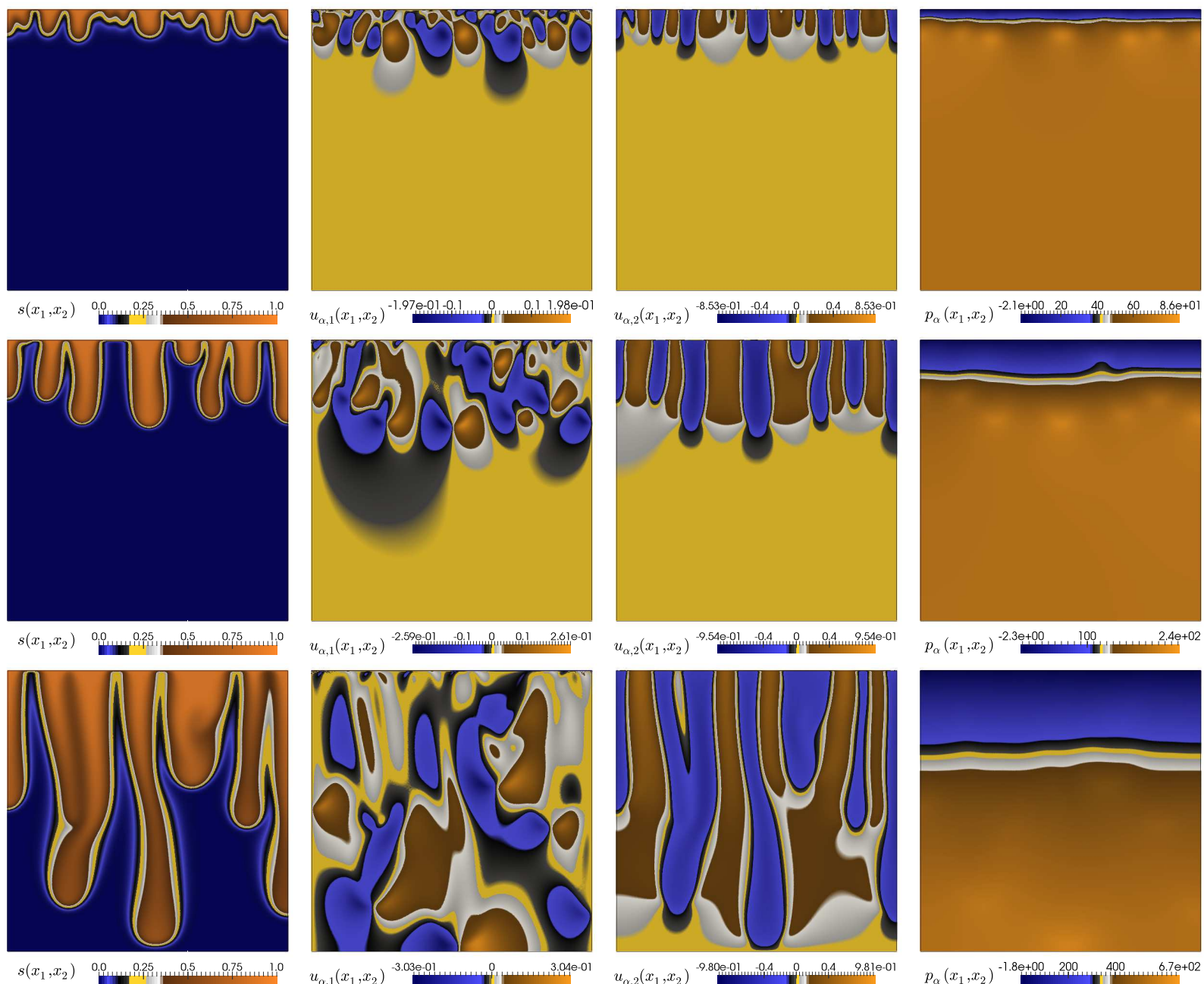

Figure 5: Example 2, test A: snapshots of the carbon dioxide dissolution into brine (left panels), distribution of horizontal and vertical velocity components (center), and pressure profiles (right column); shown at early (top row), intermediate (middle), and advanced (bottom) time instants, for a large domain with $\mathrm{Ra}=1$.

flow patterns of both cases by observing four snapshots of the concentration and vertical velocity profiles along the midline (see bottom row).

\subsection{Example 3: Terzaghi's consolidation test}

In order to validate the mixed formulation in the context of the linear poroelasticity equations presented in Section 3.3, we analyze the accuracy of the numerical scheme applied to the Terzaghi's consolidation test 31, which is a classical benchmark in geomechanics. The problem consists in determining the behavior of the excess pore pressure of the fluid when a thin porous column of height $H$ (with impermeable and rigid bottom and side walls) is compressed by a force applied instantaneously at the top. This process generates an overpressure and an associated settlement displacement $p_{\delta}$ and $d_{2}^{\delta}$, respectively. Perfect drainage is allowed at the top boundary. Under these specific conditions, separation of variables leads to the following Fourier-series solution (see also [17])

$$
d_{2}\left(x_{2}, t\right)=d_{2}^{\delta}+p_{\delta} \frac{8 H}{\pi^{2}}\left[\left(H-x_{2}\right)-\sum_{j=0}^{\infty} \frac{1}{(2 j+1)^{2}} \cos \left(\frac{(2 j+1) \pi}{2 H}\left(H-x_{2}\right)\right) \exp \left(-\frac{(2 j+1)^{2} \pi^{2} c_{c}}{4 H^{2}} t\right)\right],
$$



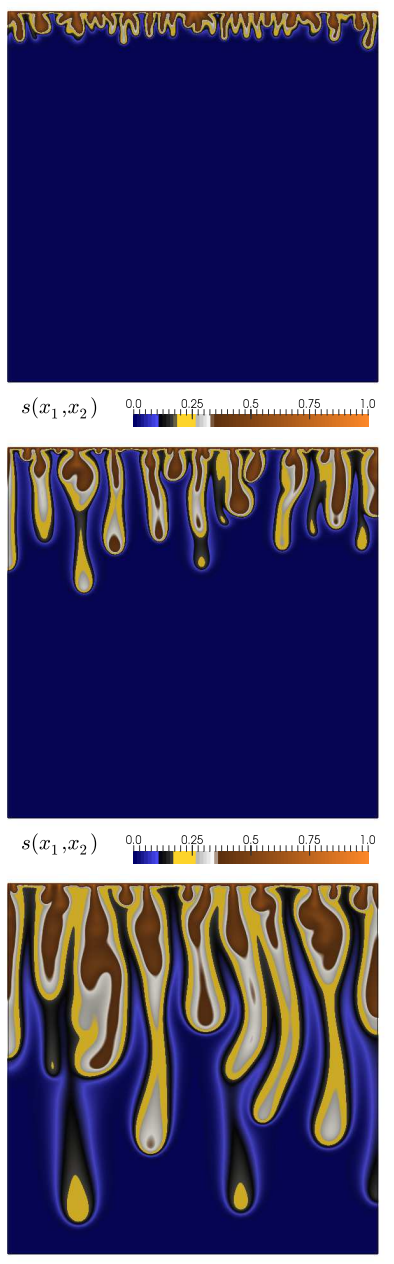

$s\left(x_{1}, x_{2}\right)$
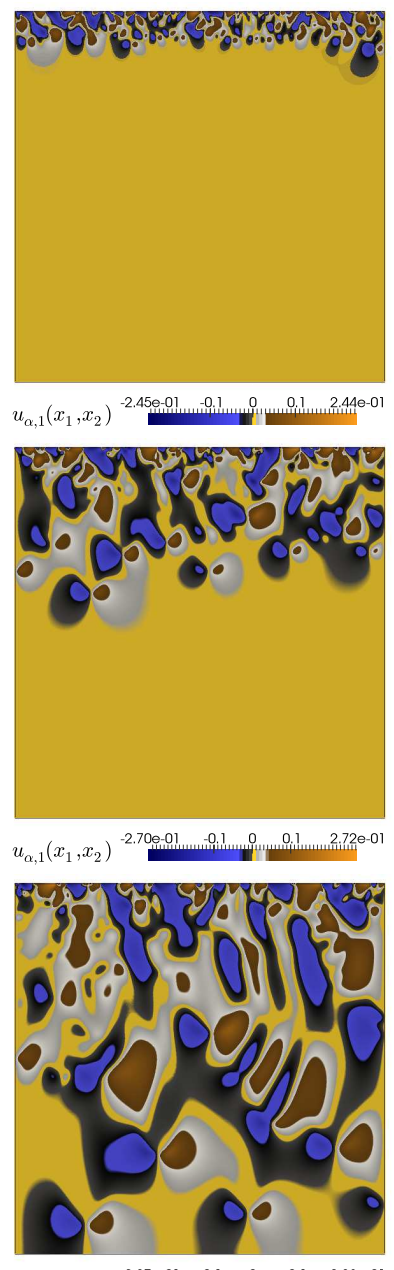

$u_{\alpha, 1}\left(x_{1}, x_{2}\right)-2.370-01$
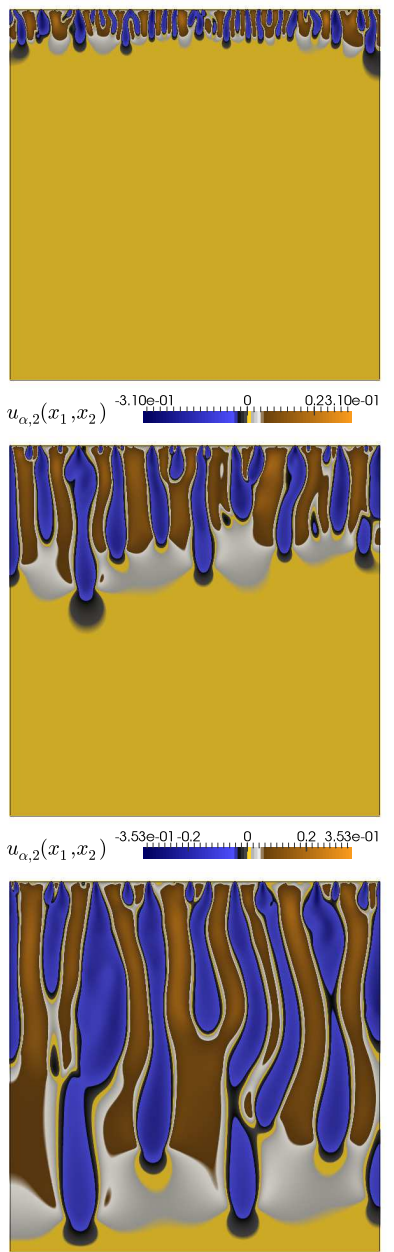

$u_{\alpha, 2}\left(x_{1}, x_{2}\right)^{-3.310-01-0.2}+0.23 .30 e-01$
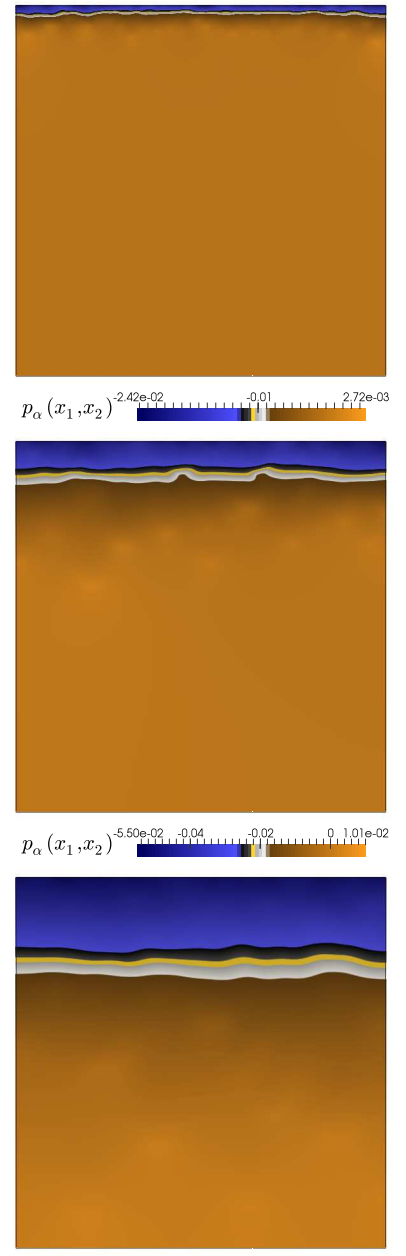

$p_{\alpha}\left(x_{1}, x_{2}\right)$

Figure 6: Example 2, test B: snapshots of the carbon dioxide dissolution into brine (left panels), horizontal and vertical velocity components (center panels) and pressure profiles (right column), displayed at three time instants, for a small domain with $\mathrm{Ra}=10000$.

$$
p\left(x_{2}, t\right)=\frac{4}{\pi} p_{\delta} \sum_{j=0}^{\infty} \frac{1}{2 j+1} \sin \left(\frac{(2 j+1) \pi}{2 H}\left(H-x_{2}\right)\right) \exp \left(-\frac{(2 j+1)^{2} \pi^{2} c_{c}}{4 H^{2}} t\right)
$$

where $c_{c}=\kappa /\left(\mu\left(c_{s}+\phi c_{f}\right)\right)$ is the consolidation coefficient, $c_{s}$ and $c_{f}$ are the solid and fluid compressibilities, respectively, $\phi$ is the porosity and $\kappa$ is the intrinsic isotropic permeability of the medium. We simulate a thin domain $\Omega=(0, W) \times(0, H)$ employing only two elements to span the width of the column and a total of 2'886 triangular elements conform the structured primal mesh. Model and discretization parameters are collected in Table 1. The process is simulated until $t=T$ and the over-pressurization of the saturated fluid decreases progressively, due to the fluid flow towards the drained top boundary. Vertical profiles of the displacement and pressure approximations at the centerline of the column are shown in Figure8, along with the consolidation profiles for adimensional quantities. They accurately match the reference analytical solutions obtained by truncating the Fourier series at $j=350$.

\subsection{Example 4: cantilever bracket with curved boundary}

We further study the behavior of the proposed scheme applied to the poroelasticity equations in (3.13). We recall that displacements are approximated with Brezzi-Douglas-Marini elements (since a higher regularity is required for the discrete bilinear forms, involving strains in terms of displacements), whereas pressure 

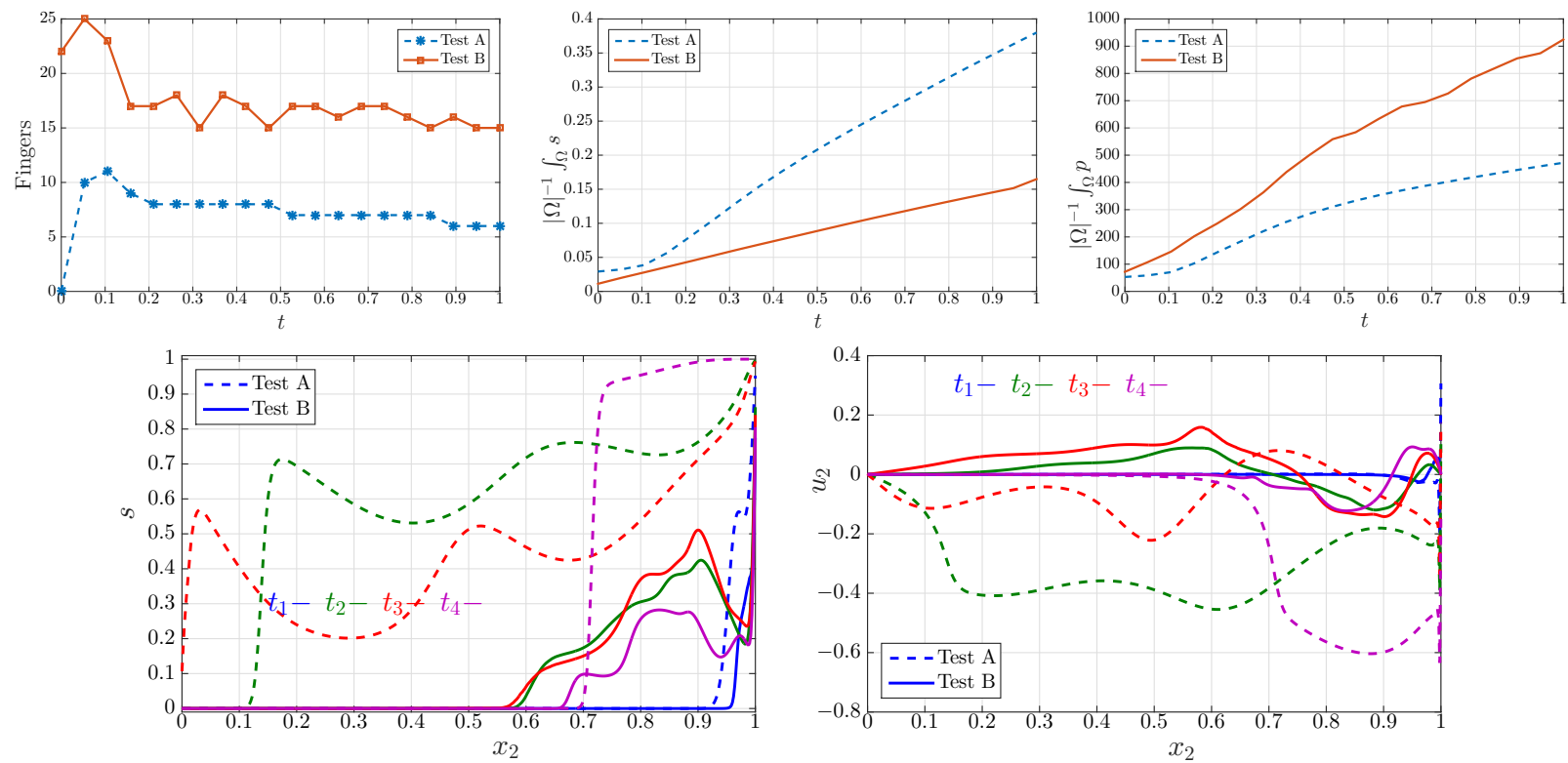

Figure 7: Example 2: evolution of the number of fingers (top left), temporal accumulation of total amount of solute (top center) and pressure (top right) for both regimes (the pressure for the test case B has been rescaled for visualization purposes), and snapshots of the $\mathrm{CO}_{2}$ concentration and vertical velocity profiles on the midline (bottom left and right, respectively).
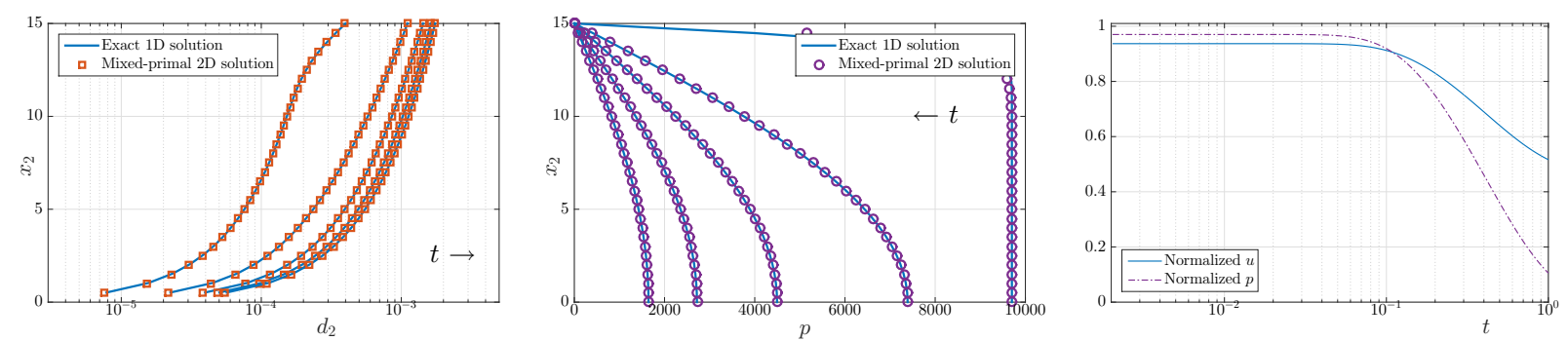

Figure 8: Example 3: analytical and numerical vertical displacements (left panel), pore pressure (center), and the degree of consolidation for Terzaghi's test. The first two plots display snapshots at time instants $t=200,400, \ldots, 1000$.

and velocity are approximated as specified at the beginning of this section. Physical parameters are outlined in Table 1, and we set $\lambda=E \nu(1+\nu)^{-1}(1-2 \nu)^{-1}, G=E /(2+2 \nu)$, where $E$ is the Young's modulus and $\nu$ the Poisson' ratio.

The domain, $\Omega$, is delimited by four curved boundaries parametrized as

$$
\begin{aligned}
& \Gamma_{1}=\left\{\omega \in[0, W]: x_{1}=\omega+\gamma \cos (\pi \omega) \sin (\pi \omega), x_{2}=-\gamma \cos (\pi \omega) \sin (\pi \omega)\right\}, \\
& \Gamma_{2}=\left\{\omega \in[0, W]: x_{1}=W+\gamma \cos (\pi \omega) \sin (\pi \omega), x_{2}=\omega-\gamma \cos (\pi \omega) \sin (\pi \omega)\right\} \text {, } \\
& \Gamma_{3}=\left\{\omega \in[W, 0]: x_{1}=\omega+\gamma \cos (\pi \omega) \sin (\pi \omega), x_{2}=W-\gamma \cos (\pi \omega) \sin (\pi \omega)\right\} \text {, } \\
& \Gamma_{4}=\left\{\omega \in[W, 0]: x_{1}=\gamma \cos (\pi \omega) \sin (\pi \omega), x_{2}=\omega-\gamma \cos (\pi \omega) \sin (\pi \omega)\right\} \text {, }
\end{aligned}
$$

and it is discretized into a primal mesh of 38'934 elements and 19'468 points.

Boundary conditions are assigned as follows: zero displacement is imposed on $\Gamma_{4}$, a unitary traction pointing downwards is applied on $\Gamma_{3}$, whereas stress free conditions are used on $\Gamma_{1} \cup \Gamma_{2}$. Free-slip velocities and constant pressure are assigned on $\partial \Omega$ (a similar configuration has been recently employed in [33], see also [35). We initialize the system with zero pressure and zero velocities, and we used a fixed timestep $\Delta t=1$. Figure 9 illustrates transient computations at instants $t=5,25,50$ for pressure, horizontal and 

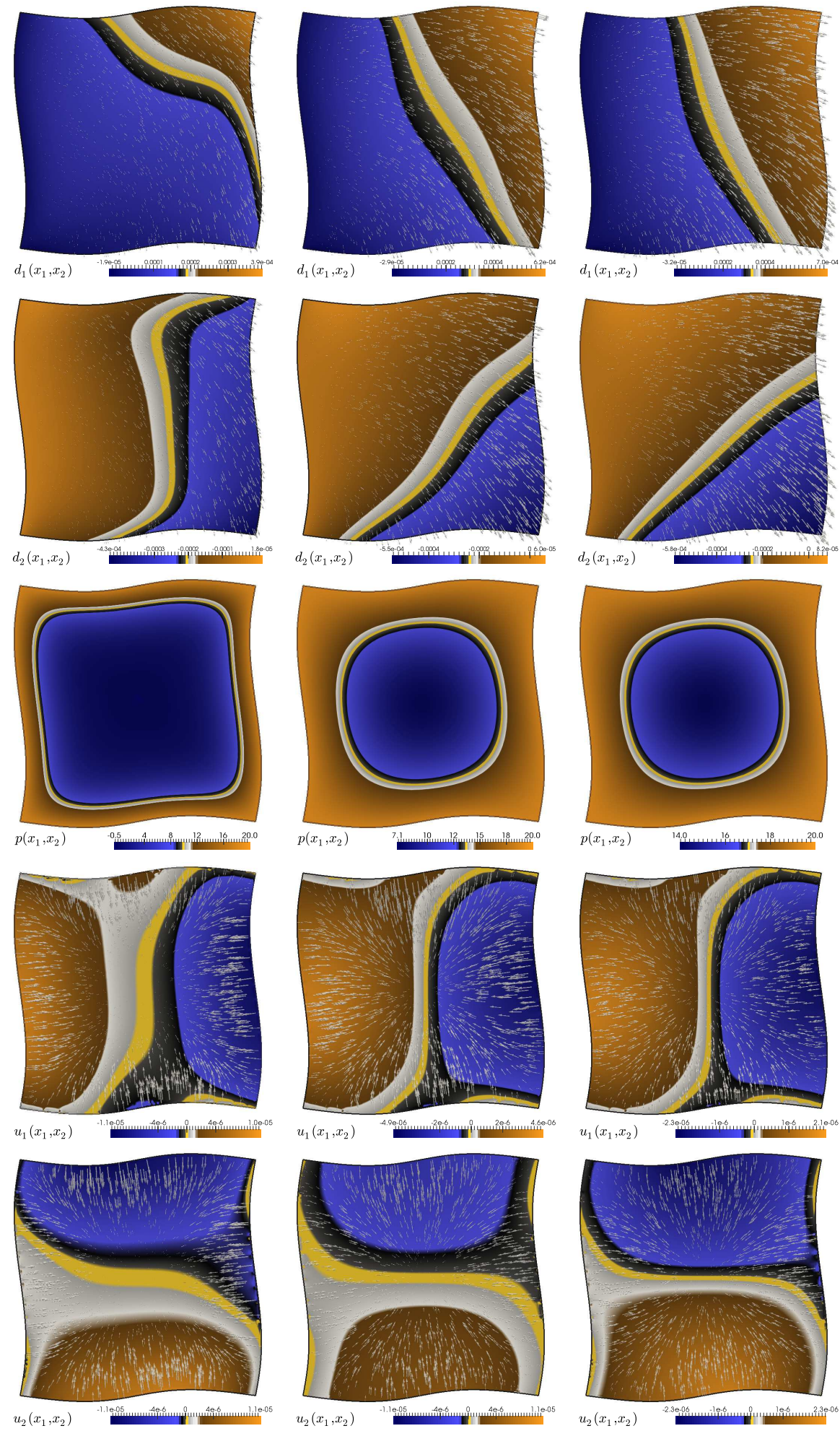

Figure 9: Example 4: snapshots of displacement components (first and second rows), pressure (third row), and velocity components and directions (fourth and last row) for the cantilever bracket problem at instants $t=5,25,50$ (left, center, and right columns, respectively). 

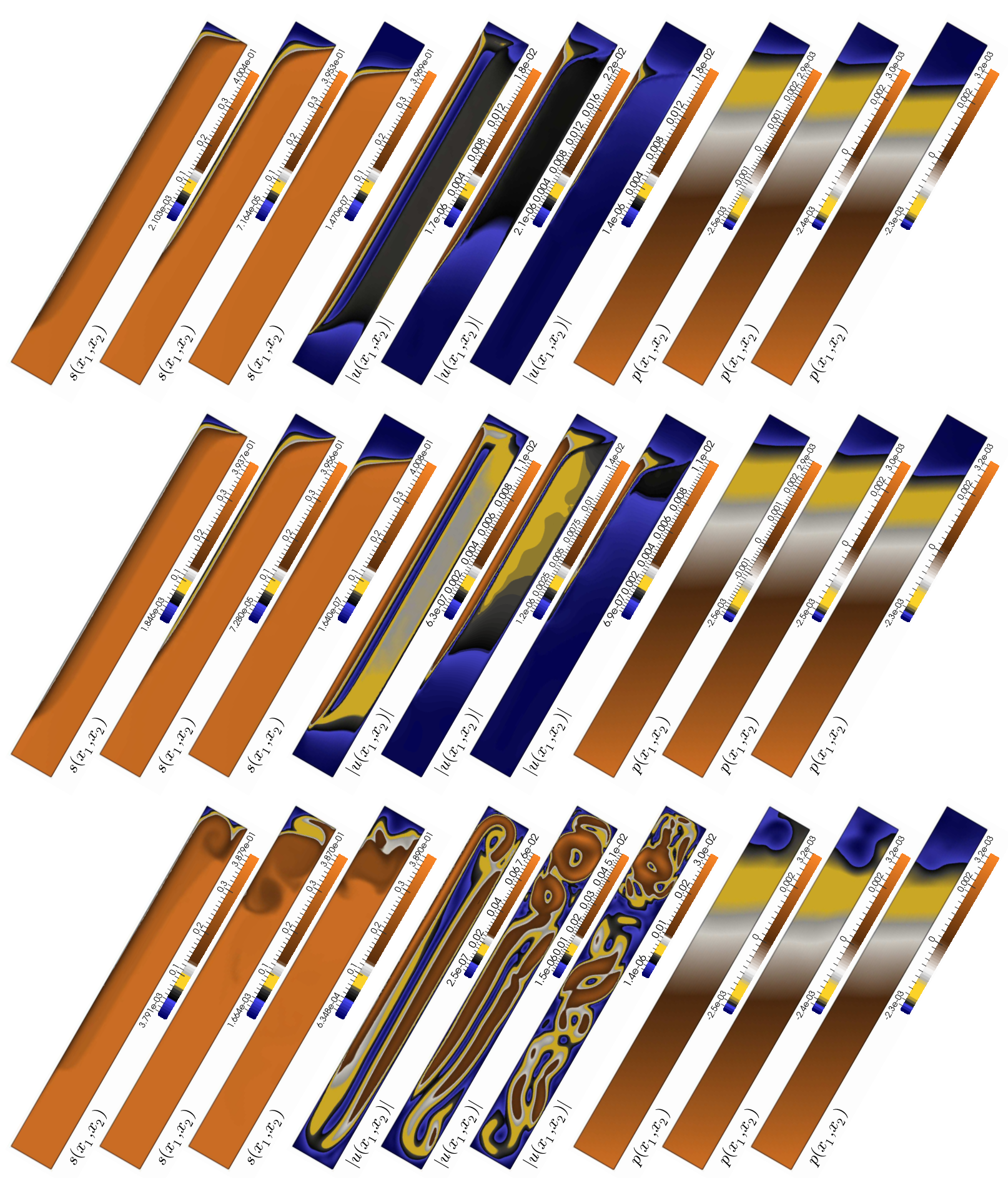

Figure 10: Example 5: snapshots at times $t=5000,10000,20000$ of the local volume fraction (left), velocity norm (middle), and pressure profiles (right) when the flow is described under Darcy (top panels), Brinkman (center), or Navier-Stokes (bottom row) regimes.

vertical displacement, and horizontal and vertical velocity, all in accordance with the results reported in [33, 35. 


\begin{tabular}{cccccccccc}
\hline$h$ & $\mathrm{e}_{\mathrm{div}}(\boldsymbol{u})$ & $r_{\text {div }}(\boldsymbol{u})$ & $\mathrm{e}_{0}(p)$ & $r_{0}(p)$ & $\mathrm{e}_{\mathrm{div}}\left(\boldsymbol{u}_{\alpha}\right)$ & $r_{\text {div }}\left(\boldsymbol{u}_{\alpha}\right)$ & $\mathrm{e}_{1}(s)$ & $r_{1}(s)$ & iter \\
\hline \hline $\mathbf{1 . 4 1 4 2}$ & $\mathbf{3 . 2 6 8 7}$ & - & $\mathbf{5 . 9 2 5 8}$ & - & $\mathbf{2 . 3 7 5 1}$ & - & $\mathbf{2 . 2 7 8 9}$ & - & 8 \\
$\mathbf{0 . 7 0 7 1}$ & $\mathbf{1 . 5 8 9 7}$ & $\mathbf{1 . 0 3 9 9}$ & $\mathbf{3 . 2 0 5 9}$ & $\mathbf{0 . 8 8 6 2}$ & $\mathbf{1 . 2 2 2 2}$ & $\mathbf{0 . 9 5 8 4}$ & $\mathbf{1 . 2 2 4 0}$ & $\mathbf{0 . 8 9 6 6}$ & 7 \\
$\mathbf{0 . 3 5 3 5}$ & $\mathbf{0 . 8 1 9 4}$ & $\mathbf{0 . 9 5 6 0}$ & $\mathbf{1 . 6 8 3 6}$ & $\mathbf{0 . 9 2 9 1}$ & $\mathbf{0 . 6 2 5 2}$ & $\mathbf{0 . 9 6 7 0}$ & $\mathbf{0 . 6 4 1 0}$ & $\mathbf{0 . 9 3 3 2}$ & $\mathbf{7}$ \\
$\mathbf{0 . 2 3 5 7}$ & $\mathbf{0 . 5 5 4 7}$ & $\mathbf{0 . 9 6 2 1}$ & $\mathbf{1 . 1 5 3 7}$ & $\mathbf{0 . 9 3 2 1}$ & $\mathbf{0 . 4 2 2 1}$ & $\mathbf{0 . 9 6 8 9}$ & $\mathbf{0 . 4 3 6 3}$ & $\mathbf{0 . 9 4 8 7}$ & $\mathbf{6}$ \\
$\mathbf{0 . 1 5 7 1}$ & $\mathbf{0 . 3 7 4 7}$ & $\mathbf{0 . 9 6 7 8}$ & $\mathbf{0 . 7 8 8 0}$ & $\mathbf{0 . 9 4 0 2}$ & $\mathbf{0 . 2 8 4 9}$ & $\mathbf{0 . 9 6 9 3}$ & $\mathbf{0 . 2 9 7 5}$ & $\mathbf{0 . 9 4 3 7}$ & 8 \\
$\mathbf{0 . 0 7 8 5}$ & $\mathbf{0 . 1 9 1 2}$ & $\mathbf{0 . 9 7 0 2}$ & $\mathbf{0 . 4 0 7 1}$ & $\mathbf{0 . 9 5 2 7}$ & $\mathbf{0 . 1 4 4 8}$ & $\mathbf{0 . 9 7 6 3}$ & $\mathbf{0 . 1 5 3 9}$ & $\mathbf{0 . 9 5 1 2}$ & 7 \\
$\mathbf{0 . 0 5 8 9}$ & $\mathbf{0 . 1 4 4 0}$ & $\mathbf{0 . 9 8 4 5}$ & $\mathbf{0 . 3 0 8 5}$ & $\mathbf{0 . 9 6 4 1}$ & $\mathbf{0 . 1 0 9 2}$ & $\mathbf{0 . 9 7 9 5}$ & $\mathbf{0 . 1 1 7 5}$ & $\mathbf{0 . 9 3 7 7}$ & 8 \\
$\mathbf{0 . 0 3 9 2}$ & $\mathbf{0 . 0 9 6 6}$ & $\mathbf{0 . 9 8 5 8}$ & $\mathbf{0 . 2 0 7 1}$ & $\mathbf{0 . 9 8 2 9}$ & $\mathbf{0 . 0 7 3 4}$ & $\mathbf{0 . 9 8 1 0}$ & $\mathbf{0 . 0 7 8 7}$ & $\mathbf{0 . 9 8 6 0}$ & 8 \\
$\mathbf{0 . 0 1 9 6}$ & $\mathbf{0 . 0 4 8 6}$ & $\mathbf{0 . 9 9 0 7}$ & $\mathbf{0 . 1 0 4 3}$ & $\mathbf{0 . 9 8 9 3}$ & $\mathbf{0 . 0 3 7 1}$ & $\mathbf{0 . 9 8 4 4}$ & $\mathbf{0 . 0 3 9 4}$ & $\mathbf{0 . 9 9 7 1}$ & 8 \\
$\mathbf{0 . 0 1 0 6}$ & $\mathbf{0 . 0 2 4 5}$ & $\mathbf{0 . 9 9 1 4}$ & $\mathbf{0 . 0 0 5 2}$ & $\mathbf{0 . 9 8 6 4}$ & $\mathbf{0 . 0 1 8 6}$ & $\mathbf{0 . 9 8 3 5}$ & $\mathbf{0 . 0 1 9 6}$ & $\mathbf{0 . 9 9 6 3}$ & 8 \\
\hline
\end{tabular}

Table 3: Example 6: convergence results and Newton iteration count for the mixed FE - primal DFVE approximation of a steady version of the coupled problem 2.3 -2.6 , computed on a sequence of uniformly refined tetrahedral partitions of $\Omega=(0, W)^{3}$.

\subsection{Example 5: sedimentation-consolidation and the Boycott effect under different flow descriptions}

The settling velocity of solid particles in a tilted vessel is significantly enhanced with respect to same process occurring in a vertical vessels. This phenomenon is known as Boycott effect: the tilted vessel offers a larger horizontal cross section that increases the area through which solid particles can settle. We assume an inclined channel of width $W$ and height $H$, forming an angle $\theta$ with the vertical axis (see Table 1 for the numerical values used in the simulations). As long as the Reynolds number is low, inertial effects are negligible and relaxation times are short, Darcy's law provides a satisfactory descriptions of the filtration velocity (the relative velocity of the fluid with respect to the solid phase). Here, we examine three models of increasing complexity: first we consider a pure Darcian model (the filtration velocity is proportional to the pressure gradient); then, we assume Stokesian flow (i.e., we add viscous effects but we do not consider the acceleration term); finally, we incorporate nonlinear convection. We remark that the dimensional analysis performed in [10], leading, in particular, to (3.9), is valid only for the first and second models considered here.

Irrespective of the specific model at hand, the hindered settling fluxes assumed in Section 3.2 lead to the formation of at least two zones of high volume-fraction gradient: at the top and left walls of the vessel a clear fluid region forms and expand downwards, whereas at the bottom and right walls a second high gradient region forms due to compaction of solid particles and expands upwards. These regions can be observed in Figure 10 where we plot the volume fraction and the velocity distributions obtained with the three different models. A spreading of the volume fraction occurs in all cases. In case of the Darcian and Stokesian models (top and center panels), the momentum equation does not contain the Lagrangian time derivative of velocity, which simply leads to the jamming of solid particles with very mild recirculation; little differences are noticed between these two cases. When a full momentum equation is used (including nonlinear convection, bottom panel of Figure 10, we observe mixing patterns initiated by local time relaxations. Figure 11 portrays a close-up to the upper part of the vessel, where the largest differences between the three cases are noticed, especially for the pressure field.

\subsection{Example 6: spatial convergence and 3-D implementation of the full multicontinuum model}

We now investigate the performance and accuracy of the mixed-primal scheme by analyzing the spatial convergence of errors computed against an analytical solution of the following stationary (but still nonlinear and fully coupled) version of the problem 2.3 $-(2.6)$, which describes the steady flow of two miscible fluids:

$$
\operatorname{div}\left(s \boldsymbol{u}_{\alpha}\right)=0
$$



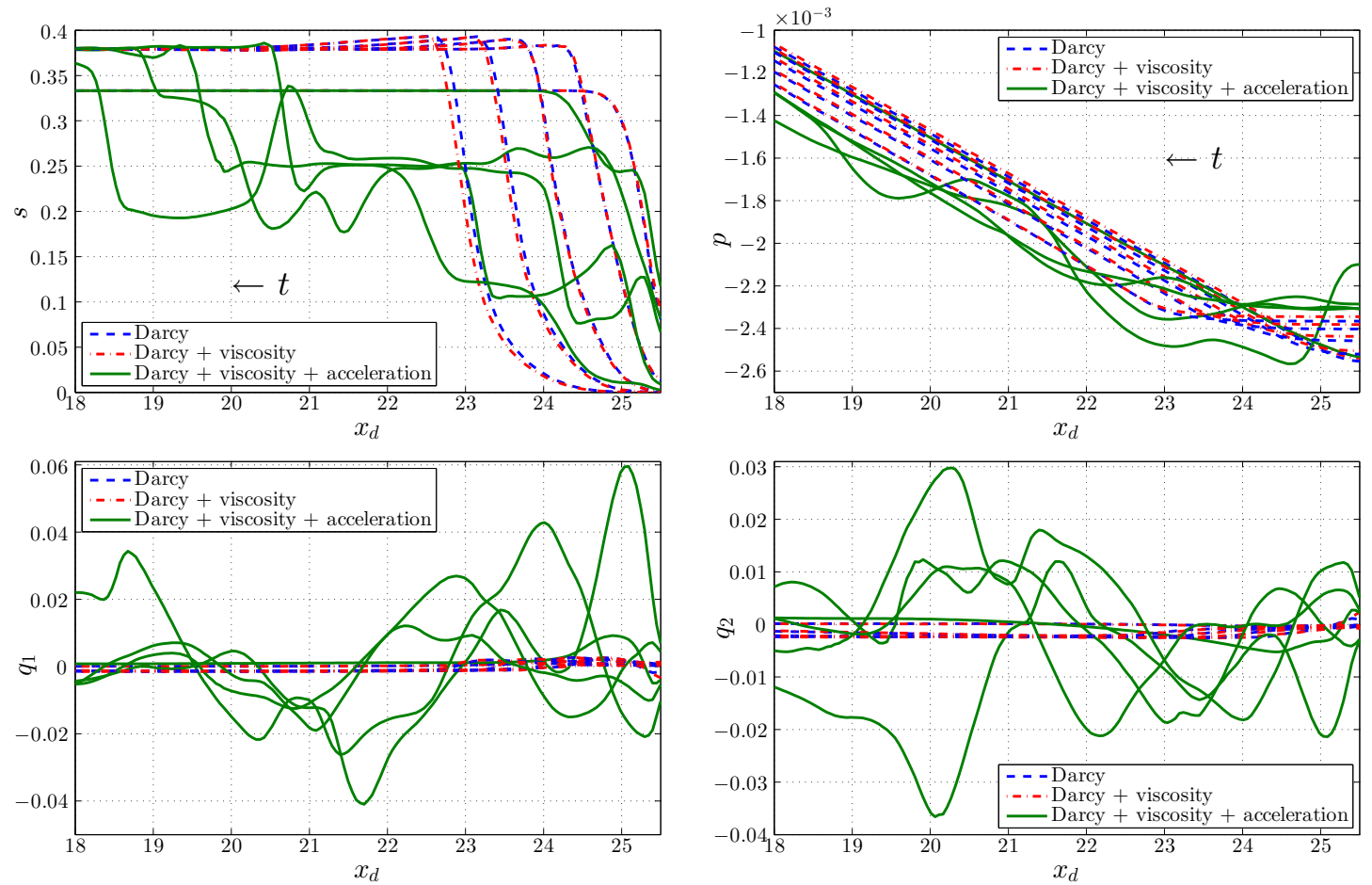

Figure 11: Example 5: zoomed profiles of the variables at the central midline of the vessel, computed at five time instants.

$$
\begin{array}{r}
\operatorname{div}\left(s \boldsymbol{u}_{\alpha} \otimes \boldsymbol{u}_{\alpha}\right)-\operatorname{div} \boldsymbol{\sigma}_{\alpha}-\frac{1}{\rho_{\alpha}} p_{\alpha}(p, s) \nabla s-D(s)\left(\frac{\rho(s) \boldsymbol{u}-\rho_{\alpha} s \boldsymbol{u}_{\alpha}}{\rho_{\beta}(1-s)}-\boldsymbol{u}_{\alpha}\right)-s \boldsymbol{g}=\boldsymbol{k}_{\alpha}, \\
\operatorname{div}(\rho(s) \boldsymbol{u})=0, \\
\rho(s) \operatorname{div}(\boldsymbol{u} \otimes \boldsymbol{u})-\operatorname{div} \boldsymbol{\sigma}+p_{c}(s) \nabla s-\rho(s) \boldsymbol{g}=\boldsymbol{k} .
\end{array}
$$

We proceed to manufacture the following exact solutions to this problem defined on a cube $\Omega=(0, W)^{3}$ :

$$
\begin{aligned}
& s(\boldsymbol{x})=\pi \exp \left(-\left(x_{1}-\frac{1}{2}\right)\left(x_{2}-\frac{1}{2}\right)\left(x_{3}-\frac{1}{2}\right)\right)+a, p(\boldsymbol{x})=\sin \left(\pi\left(x_{1}-\frac{1}{2}\right)\right) \sin \left(\pi\left(x_{2}-\frac{1}{2}\right)\right) \sin \left(\pi\left(x_{3}-\frac{1}{2}\right)\right), \\
& \boldsymbol{u}(\boldsymbol{x})=\frac{1}{\rho_{\beta}+\left(\rho_{\beta}-\rho_{\alpha}\right) s(\boldsymbol{x})}\left(\begin{array}{c}
\cos \left(\pi\left(x_{1}-\frac{1}{2}\right)\right) \sin \left(\pi\left(x_{2}-\frac{1}{2}\right)\right) \sin \left(\pi\left(x_{3}-\frac{1}{2}\right)\right) \\
\sin \left(\pi\left(x_{1}-\frac{1}{2}\right)\right) \cos \left(\pi\left(x_{2}-\frac{1}{2}\right)\right) \sin \left(\pi\left(x_{3}-\frac{1}{2}\right)\right) \\
-2 \sin \left(\pi\left(x_{1}-\frac{1}{2}\right)\right) \sin \left(\pi\left(x_{2}-\frac{1}{2}\right)\right) \cos \left(\pi\left(x_{3}-\frac{1}{2}\right)\right)
\end{array}\right), \\
& \boldsymbol{u}_{\alpha}(\boldsymbol{x})=\frac{1}{s(\boldsymbol{x})}\left(\begin{array}{c}
\cos \left(x_{1}-\frac{1}{2}\right) \sin \left(x_{2}-\frac{1}{2}\right) \sin \left(x_{3}-\frac{1}{2}\right) \\
\sin \left(x_{1}-\frac{1}{2}\right) \cos \left(x_{2}-\frac{1}{2}\right) \sin \left(x_{3}-\frac{1}{2}\right) \\
-2 \sin \left(x_{1}-\frac{1}{2}\right) \sin \left(x_{2}-\frac{1}{2}\right) \cos \left(x_{3}-\frac{1}{2}\right)
\end{array}\right)
\end{aligned}
$$

and we choose the constitutive relationships and the saturation-dependent coefficients in the form

$$
\rho(s)=\rho_{\beta}+\left(\rho_{\beta}-\rho_{\alpha}\right) s, \quad D(s)=s^{m}, \quad p_{\alpha}(p, s)=\frac{p-(1-s) p_{c}(s)}{2 s-1}, \quad p_{c}(s)=\left(\frac{s-b}{1-b}\right)^{-1 / m},
$$

where the parameters are taken as specified in Table 1. The forcing terms $\boldsymbol{k}, \boldsymbol{k}_{\alpha}$ and the boundary data are imposed according to the exact solutions above. The convergence history displayed in Table 3 shows a first order convergence for the total and phase velocities, pressure and saturation of phase $\alpha$ in their relevant norms. The approximate fields computed after seven steps of nonuniform refinement are provided in Figure 12 


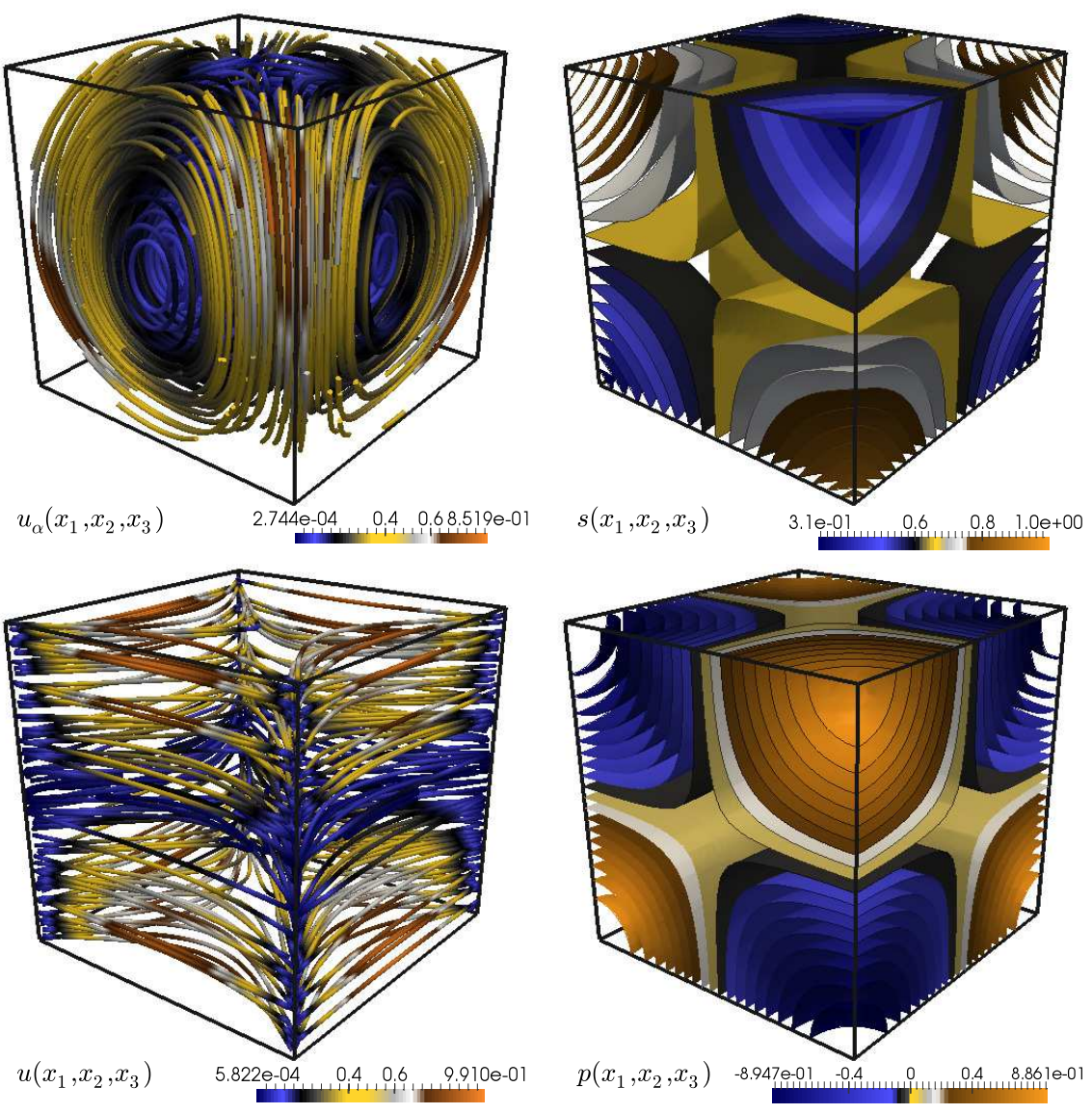

Figure 12: Example 6: approximate solutions for the two-phase Brinkman flow corresponding to the steady counterpart of 2.3. -2.6 computed on a tetrahedral mesh of 228690 primal cells and 39304 vertices. We depict velocity streamlines, and contour plots of pressure and saturation profiles.

\subsection{Example 7: two-phase flow in an inclined cylinder}

Let us again consider the Boycott effect as in Example 5 above, but now focusing on the patterns obtained in the case of a dense granular material. The governing equations now correspond to the full model in (2.3)2.6. The domain of interest is a 3-D cylinder of height $H$ and radius $R$, with longitudinal axis tilted by an angle $\theta$ with respect to the vertical. It is discretized into an unstructured primal mesh of $48^{\prime} 361$ vertices and 267'297 tetrahedra. The interface drag coefficient in 2.2 is assumed as in the Carman-Kozeny's law for granular material (see e.g. [29]), i.e., $D(s)=\frac{150 \nu_{\beta} s^{2}}{j^{2}(1-s)}$, where $j$ is the particle size.

In a first test (A), we assume no-flux boundary conditions for the volume fraction, and no-slip conditions are set on the whole boundary for phase $\alpha$ and for the barycentric velocity, that is, we model the batch sedimentation case. The mixture is initially homogeneous, with $s=s_{0}^{A}$ on the whole domain, and at rest. Phase $\beta$ will represent an immiscible fluid (e.g. water) with properties $\rho_{\beta}^{A}, \mu_{\beta}^{A}$ as specified in Table 1. A fixed time step $\Delta t^{A}=0.02$ is employed. The approximate solutions at the final time are displayed in Figure 13 and are in qualitative agreement with similar simulations presented in [30].

In a second test (B) we simulate the interaction of the solid granular phase $\alpha$ (having the same properties as in test A) with a gaseous phase $\beta$ (considered also incompressible at low Mach numbers) with properties $\rho_{\beta}^{B}, \mu_{\beta}^{B}$, also specified in Table 1. The vessel is initially filled by a resting homogeneous mixture with solid fraction $s_{0}^{B}$. A constant inflow of gas with velocity magnitude $q_{\beta}$ is injected in the vessel at the bottom boundary, whereas a constant pressure of the solid phase is assumed at the top of the cylinder, where both gas and solid material are allowed to leave the vessel. We assume that the maximum packing of the solid 

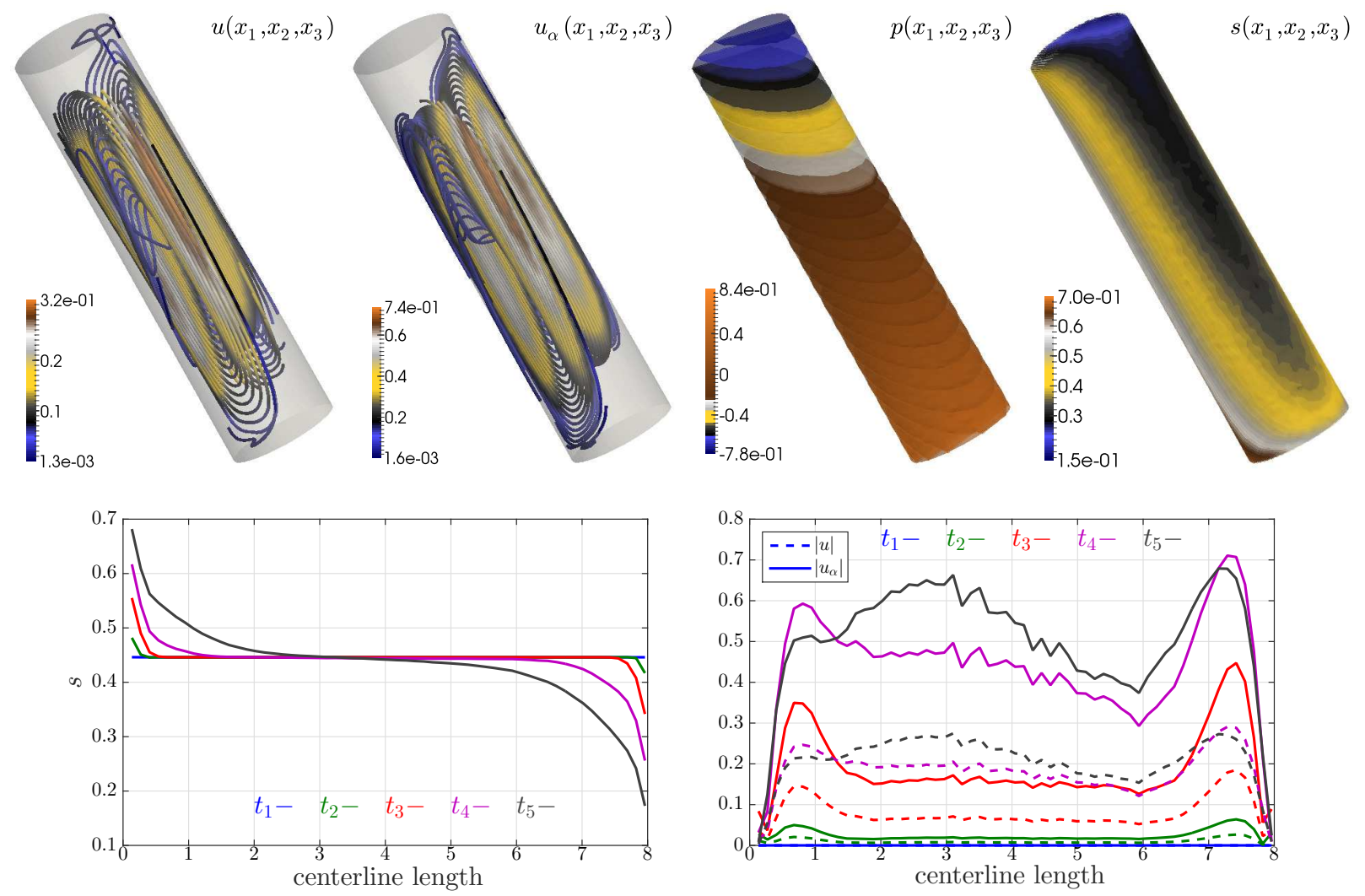

Figure 13: Example 7, test A. Top plots from left to right: mixed-primal approximations of total and phasic velocity streamlines, pressure iso-surfaces, and solids volume fraction field for the two-phase sedimentation process in a tilted cylinder. Snapshots correspond to an advanced (adimensional) time $t=200$. The bottom panels show five profiles of the fields at the centerline of the cylinder.

phase is $s_{\max }$. The timestep is now $\Delta t=0.0001$. Figure 14 portrays the numerical results corresponding to test B. As expected, the underlying mechanisms of granular-gas interactions yield very different mixing patterns from those observed in its fluid-based sedimentation counterpart. In particular, we observe the formation of bubble-like structures and flow fragmentation, in contrast to simple compaction and formation of layers perpendicular to the gravity direction as those evidenced in test A.

\section{Concluding remarks}

We have presented a novel discretization scheme that is tailored to simulate general multicontinuum models and combines a mixed FE method for the momentum equations (or derivation of thereof) with a primal DFVE method for the transport equations. The method is devised for a system of equations describing mass and momentum conservations of the mixture and of one of the phases. This allows us to readily derive discretization schemes to deal with models of increasing complexity and to study virtually any physical multicontinuum systems. Moreover, the general (archetypal) models for which the method is derived can be seen as a generalization that extends many particular multiphase models and can applied to problems in which the assumptions leading to the latter are not satisfied.

The main features of the discretization scheme are local conservation, satisfaction of discrete maximum principles and divergence free properties, arbitrary accuracy of fluxes, controlled numerical diffusion, and a Petrov-Galerkin structure that is appropriate to derive error estimates in the energy norm. In addition, the method is developed for unstructured triangular and tetrahedral meshes, it is suitable 

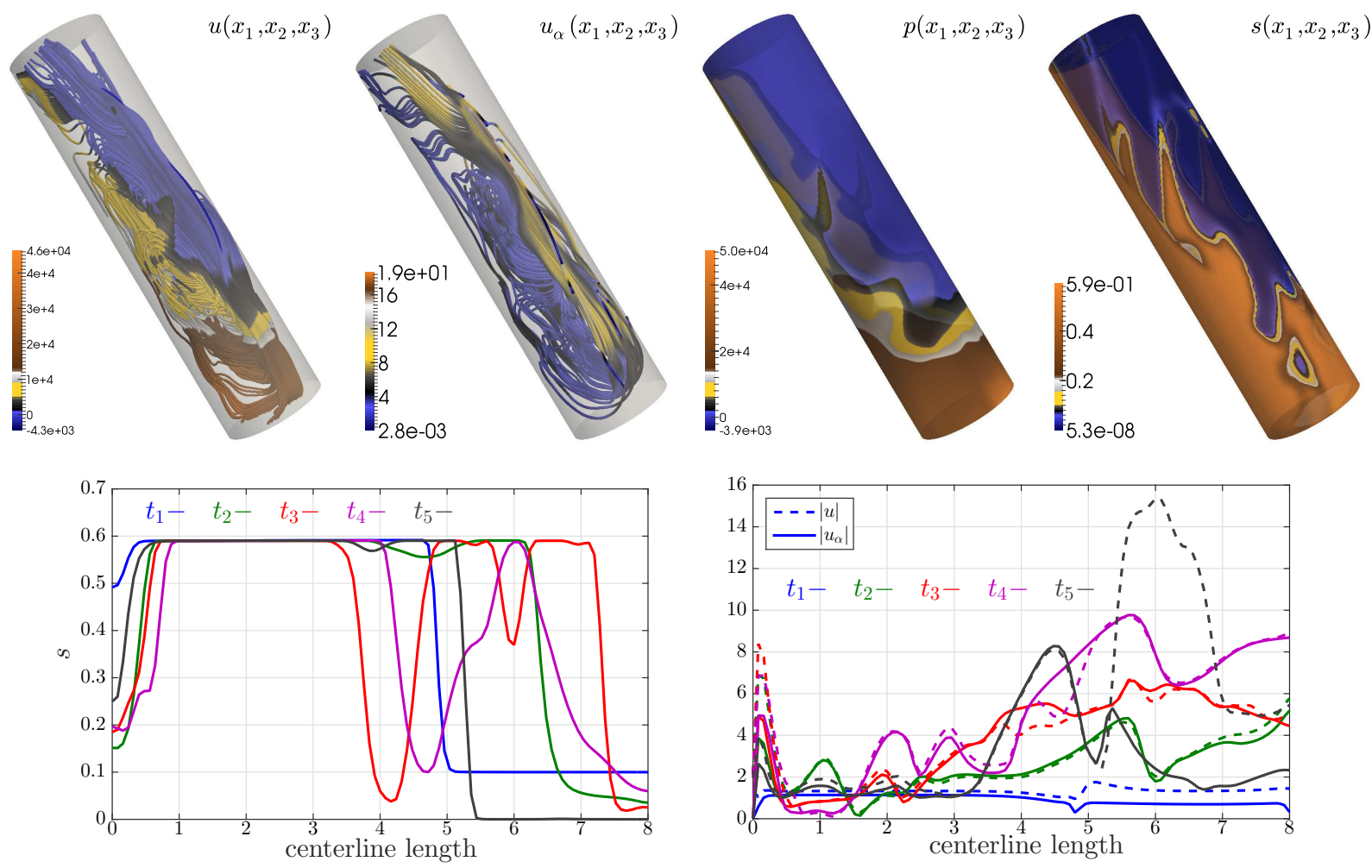

Figure 14: Example 7, test B. Top plots from left to right: mixed-primal approximations of total and phasic velocity streamlines, pressure profiles, and solids volume fraction field for the two-phase sedimentation process in a tilted cylinder. Snapshots correspond to an advanced (adimensional) time $t=15$. The bottom panels show five profiles of the fields at the centerline of the cylinder.

to handle very complex geometries, and guarantees coupling stability by an exact Newton linearization.

An extensive set of numerical examples has been presented to validate the spatiotemporal accuracy of the method that has proven to have an optimal convergence rate in the sense that errors decay with the same order as the corresponding interpolators. The numerical results also demonstrate the robustness of the method, which has been successfully applied to diverse physical phenomena such as density fingering driven by gravity instability, Terzaghi's consolidation test, deformation of a cantilever bracket, Boycott effects during sedimentation. In particular, for the Boycott problem we have also shown that the numerical method can handle well the most complex physical model, which includes a fully coupled system of two mass and two momentum equations. Beside the quality of the discretization schemes, the accuracy of the numerical method relies on the implicit and monolithic nature of the coupled solver that yields stable approximations of all fields in all test cases and configurations.

Several strategies can be implemented to further extend and possibly improve our method. For instance, the stability of different coupling strategies (such as splitting algorithms) can be assessed by an automatic thresholding of Jacobian blocks; smart solvers can be devised using decoupled schemes as preconditioners for the strongly coupled system; the two momentum equations can be written as mixed problems in terms of the vorticity field; augmented formulations can be introduced to permit a more flexible choice of FE spaces; and a posteriori error indicators can be applied to devise adaptive strategies to solve the transport equations. Other interesting variants of the present method that can be investigated include fully mixed and augmented FE discretizations of (2.3)-(2.6), and primal formulations of such a system that employ pure DFVE approximations of all fields. 
Finally, we remark that, even if we have concentrated mainly on hydrodynamic coupling, the system considered here displays many similarities with problems arising when modeling multi-component materials and our method can be readily extended to those applications.

\section{Acknowledgment}

Financial support for this research was provided by the Swiss National Science Foundation through the grant SNSF PP00P2-144922.

\section{References}

[1] B. Amaziane, M. JuraK, And A. ŽGaljić KeKo, An existence result for a coupled system modeling a fully equivalent global pressure formulation for immiscible compressible two-phase flow in porous media, J. Differ. Equ. (2011), 250(3):16851718 .

[2] T.B. Anderson and R. Jackson, A fluid mechanical description of fluidized beds, I\&EC Fundam. (1967) 6:527-534.

[3] T. Arbogast, The existence of weak solutions to single porosity and simple dual-porosity models of two-phase incompressible flow, Nonlinear Anal. (1992), 19(11):1009-1031.

[4] L. Bergamaschi, S. Mantica, And G. Manzini, A mixed finite element-finite volume formulation of the black-oil model, SIAM J. Sci. Comput. (1998), 20:970-997.

[5] M.A. Biot, Thermoelasticity and irreversible thermodynamics, J. Appl. Phys. (1956), 27:240-253.

[6] R.M. Bowen And A.C. Eringen, Theory of Mixtures, Part I. Continuum Physics III. Academic Press, New York (1976).

[7] R. Bürger, S. Kumar, And R. Ruiz-Baier, Discontinuous finite volume element discretization for coupled flow-transport problems arising in models of sedimentation, J. Comput. Phys. (2015), 299:446-471.

[8] R. Bürger, C. Liu, AND W.L. Wendland, Existence and stability for mathematical models of sedimentationconsolidation processes in several space dimensions, J. Math. Anal. Appl. (2001), 264:288-310.

[9] R. Bürger, R. Ruiz-Baier, And H. Torres, A stabilized finite volume element formulation for sedimentationconsolidation processes, SIAM J. Sci. Comput. (2012), 34:B265-B289.

[10] R. Bürger, W.L. Wendland, AND F. CONCHA, Model equations for gravitational sedimentation-consolidation processes, ZAMM Z. Angew. Math. Mech. (2000), 80:79-92.

[11] C. Calgaro, E. Creusé, and T. Goudon, An hybrid finite volume-finite element method for variable density incompressible flows, J. Comput. Phys. (2008), 227(9):4671-4696.

[12] C. Cancìs, I.S. Pop, AND M. VohralíK, An a posteriori error estimate for vertex-centered finite volume discretizations of immiscible incompressible two-phase flow, Math. Comp. (2014), 83:153-188.

[13] O. Coussy, Poromechanics, John Wiley \& Sons Ltd., Chichester (2004).

[14] M. Cui And X. YE, Unified analysis of finite volume methods for the Stokes equations, SIAM J. Numer. Anal. (2010), 48:824-839.

[15] C. Dawson, S. Sun, and M.F. Wheeler, Compatible algorithms for coupled flow and transport, Comput. Methods Appl. Mech. Engrg. (2004), 193:2565-2580.

[16] A. ERn And J.-L. Guermond, Theory and practice of finite elements, Applied Mathematical Sciences vol. 159, SpringerVerlag, New York (2004).

[17] M. Ferronato, N. Castelletto, and G. Gambolati, A fully coupled 3-D mixed finite element model of Biot consolidation, J. Comput. Phys. (2010), 229:4813-4830.

[18] C. Galusinski And M. SAad, On a degenerate parabolic system for compressible, immiscible, two-phase flows in porous media, Adv. Diff. Eqns. (2004), 11-12:1235-1278.

[19] F. Gao, Y. Yuan, and D. Yang, An upwind finite-volume element scheme and its maximum-principle-preserving property for nonlinear convection-diffusion problem, Int. J. Numer. Methods Fluids (2008), 56:2301-2320.

[20] G.N. GaticA, A Simple Introduction to the Mixed Finite Element Method. Theory and Applications, Springer-Verlag, Berlin (2014). 
[21] S. Geiger, S. Roberts, S.K. MatthäI, C. Zoppou, And A. Burri, Combining finite element and finite volume methods for efficient multiphase flow simulations in highly heterogeneous and structurally complex geologic media, Geofluids (2004), 4:284-299.

[22] C. Geuzaine And J.F. Remacle, Gmsh: a three-dimensional finite element mesh generator with built-in pre- and postprocessing facilities, Int. J. Numer. Methods Engrg. (2009), 79:1309-1331.

[23] J. Könnö and R. Stenberg, H(div)-conforming finite elements for the Brinkman problem, Math. Models Methods Appl. Sci. (2011), 21:2227-2248.

[24] K. Kumar, I.S. POP, AND F.A. RADU, Convergence analysis of mixed numerical schemes for reactive flow in a porous medium, SIAM J. Numer. Anal. (2013), 51:2283-2308.

[25] S. KumAR, A mixed and discontinuous Galerkin finite volume element method for incompressible miscible displacement problems in porous media, Numer. Methods Partial Diff. Eqns. (2012), 28:1354-1381.

[26] S. KumAR AND R. RuIz-BAIER, Equal order discontinuous finite volume element methods for the Stokes problem, J. Sci. Comput. (2015), 65:956-978.

[27] R. Künze And I. LunAti, An adaptive multiscale method for density-driven instabilities, J. Comput. Phys. (2012), 231:5557-5570.

[28] M. OhlBerger, Convergence of a mixed finite element-finite volume method for the two phase flow in porous media, East-West J. Numer. Math. (1997), 5:183-210.

[29] M. Pailha And O. Pouliquen, A two-phase flow description of the initiation of underwater granular avalanches, J. Fluid Mech. (2009), 633:115-135.

[30] R. RuIZ-BAIER AND H. TORRES, Numerical solution of a multidimensional sedimentation problem using finite volumeelements, Appl. Numer. Math. (2015), 95:280-291.

[31] K. Terzaghi, Theoretical Soil Mechanics, Wiley, New York (1943).

[32] J. Wang AND X. YE, New finite element methods in computational fluid dynamics by H(div) elements, SIAM J. Numer. Anal. (2007), 45:1269-1286.

[33] M.F. Wheeler, G. Xue, AND I. Yotov, Coupling multipoint flux mixed finite element methods with continuous Galerkin methods for poroelasticity, Comput. Geosci. (2014), 18:57-75.

[34] Y. Xie, C.T. Simmons, A.D. Werner, AND J.D. WARD, Effect of transient solute loading on free convection in porous media, Water Res. Research (2010), 46 W11511:1-16.

[35] S.-Y. YI, Convergence analysis of a new mixed finite element method for Biot's consolidation model, Numer. Methods Partial Diff. Eqns. (2014), 30:1189-1210. 